\title{
Semicanonical bases and preprojective algebras II: A multiplication formula
}

\author{
Christof Geiss, Bernard Leclerc and Jan Schröer
}

\begin{abstract}
Let $\mathfrak{n}$ be a maximal nilpotent subalgebra of a complex symmetric Kac-Moody Lie algebra. Lusztig has introduced a basis of $U(\mathfrak{n})$ called the semicanonical basis, whose elements can be seen as certain constructible functions on varieties of nilpotent modules over a preprojective algebra of the same type as $\mathfrak{n}$. We prove a formula for the product of two elements of the dual of this semicanonical basis, and more generally for the product of two evaluation forms associated to arbitrary modules over the preprojective algebra. This formula plays an important rôle in our work on the relationship between semicanonical bases, representation theory of preprojective algebras, and Fomin and Zelevinsky's theory of cluster algebras. It was inspired by recent results of Caldero and Keller.
\end{abstract}

\section{Introduction and main result}

\subsection{Semicanonical bases}

Let $\Lambda$ be the preprojective algebra associated to a connected quiver $Q$ without loops. This is the associative algebra

$$
\Lambda=\mathbb{C} \bar{Q} /\left\langle\sum_{a \in Q_{1}}(\bar{a} a-a \bar{a})\right\rangle,
$$

where $\bar{Q}$ denotes the double of $Q$ and $Q_{1}$ is the set of arrows of $Q$ (see, e.g., [Rin98, GLS05a]). Recall that $\bar{Q}$ is obtained from $Q$ by inserting for each arrow $a: i \rightarrow j$ in $Q$ a new arrow $\bar{a}: j \rightarrow i$. The algebra $\Lambda$ is independent of the orientation of $Q$, and it is finite-dimensional (and selfinjective) if and only if the underlying graph $\Delta$ of $Q$ is a simply laced Dynkin diagram, i.e. if $\Delta \in\left\{\mathrm{A}_{n}(n \geqslant 1)\right.$, $\left.\mathrm{D}_{n}(n \geqslant 4), \mathrm{E}_{n}(n=6,7,8)\right\}$. In the sequel, all $\Lambda$-modules are assumed to be finite-dimensional nilpotent left modules. We denote by $I$ the set of vertices of $Q$, and by $\Lambda_{\mathbf{d}}$ the affine variety of nilpotent $\Lambda$-modules with dimension vector $\mathbf{d}=\left(d_{i}\right)_{i \in I}$. The reductive group $\mathrm{GL}_{\mathbf{d}}=\prod_{i \in I} \mathrm{GL}_{d_{i}}(\mathbb{C})$ acts on $\Lambda_{\mathbf{d}}$ by base change.

Let $\mathfrak{n}$ be a maximal nilpotent subalgebra of a complex Kac-Moody Lie algebra $\mathfrak{g}$ of type $\Delta$. Lusztig [Lus91, Lus00] proved that the enveloping algebra $U(\mathfrak{n})$ is isomorphic to

$$
\mathcal{M}=\bigoplus_{\mathbf{d} \in \mathbb{N}^{I}} \mathcal{M}(\mathbf{d})
$$

where the $\mathcal{M}(\mathbf{d})$ are certain vector spaces of $\mathrm{GL}_{\mathbf{d}}$-invariant constructible functions on the affine varieties $\Lambda_{\mathbf{d}}$ (see $\S 2.1$ for the definition of $\mathcal{M}(\mathbf{d})$ ). This yields a new basis $\mathcal{S}$ of $U(\mathfrak{n})$ indexed by the irreducible components of the varieties $\Lambda_{\mathbf{d}}$, called the semicanonical basis [Lus00].

Received 29 March 2006, accepted in final form 21 February 2007.

2000 Mathematics Subject Classification 14M99, 16G20, 17B35, 17B67, $20 \mathrm{G} 05$.

Keywords: universal enveloping algebra, semicanonical basis, preprojective algebra, flag variety, composition series.

This journal is (C) Foundation Compositio Mathematica 2007. 


\section{Geiss, B. Leclerc And J. Schröer}

Let $\mathcal{M}^{*}$ be the graded dual of $\mathcal{M}$. A multiplication on $\mathcal{M}^{*}$ is defined via the natural comultiplication of the Hopf algebra $U(\mathfrak{n}) \cong \mathcal{M}$. In [GLS05a] we considered the basis $\mathcal{S}^{*}$ of $\mathcal{M}^{*}$ dual to the semicanonical basis of $\mathcal{M}$, and began to study its multiplicative properties.

\subsection{The $\delta$-stratification}

For a $\Lambda$-module $x \in \Lambda_{\mathrm{d}}$ define the evaluation form $\delta_{x}: \mathcal{M} \rightarrow \mathbb{C}$ which maps a constructible function $f \in \mathcal{M}(\mathbf{d})$ to $f(x)$. Define

$$
\langle x\rangle:=\left\{y \in \Lambda_{\mathbf{d}} \mid \delta_{y}=\delta_{x}\right\} .
$$

This is a constructible subset of $\Lambda_{\mathbf{d}}$ since $\mathcal{M}(\mathbf{d})$ is a finite-dimensional space of constructible functions on $\Lambda_{\mathbf{d}}$. For the same reason we can choose a finite set $R(\mathbf{d}) \subset \Lambda_{\mathbf{d}}$ such that

$$
\Lambda_{\mathbf{d}}=\bigsqcup_{x \in R(\mathbf{d})}\langle x\rangle .
$$

Each irreducible component $Z$ of $\Lambda_{\mathbf{d}}$ has a unique stratum $\langle x\rangle \cap Z$ containing a dense open subset of $Z$, and the points of this stratum are called the generic points of $Z$. We can then reformulate the definition of $\mathcal{S}^{*}$ as follows: the element $\rho_{Z}$ of $\mathcal{S}^{*}$ labeled by $Z$ is equal to $\delta_{x}$ for a generic point $x$ of $Z$.

The aim of this paper is to prove a general formula for the product of two arbitrary evaluation forms $\delta_{x}$.

\subsection{Extensions}

Let $x^{\prime} \in \Lambda_{\mathbf{d}^{\prime}}, x^{\prime \prime} \in \Lambda_{\mathbf{d}^{\prime \prime}}$, and $\mathbf{e}:=\mathbf{d}^{\prime}+\mathbf{d}^{\prime \prime}$. For a constructible $\mathrm{GL}_{\mathbf{e}}$-invariant subset $S \subseteq \Lambda_{\mathbf{e}}$ we consider

$$
\operatorname{Ext}_{\Lambda}^{1}\left(x^{\prime}, x^{\prime \prime}\right)_{S}=\left\{\left[0 \rightarrow x^{\prime \prime} \rightarrow y \rightarrow x^{\prime} \rightarrow 0\right] \in \operatorname{Ext}_{\Lambda}^{1}\left(x^{\prime}, x^{\prime \prime}\right) \backslash\{0\} \mid y \in S\right\} .
$$

This is a constructible $\mathbb{C}^{*}$-invariant subset of the quasi-affine space $\operatorname{Ext}_{\Lambda}^{1}\left(x^{\prime}, x^{\prime \prime}\right) \backslash\{0\}$, see $\S 5$.5. Thus, we may consider the constructible subset $\mathbb{P} \operatorname{Ext}_{\Lambda}^{1}\left(x^{\prime}, x^{\prime \prime}\right)_{S}:=\operatorname{Ext}_{\Lambda}^{1}\left(x^{\prime}, x^{\prime \prime}\right)_{S} / \mathbb{C}^{*}$ of the projective space $\mathbb{P} \operatorname{Ext}_{\Lambda}^{1}\left(x^{\prime}, x^{\prime \prime}\right)$. For a constructible subset $U$ of a complex variety let $\chi(U)$ be its (topological) Euler characteristic with respect to cohomology with compact support. For the empty set $\emptyset$ we set $\chi(\emptyset)=0$. Note that we have, by the additivity of $\chi$,

$$
\sum_{x \in R(\mathbf{e})} \chi\left(\mathbb{P} \operatorname{Ext}_{\Lambda}^{1}\left(x^{\prime}, x^{\prime \prime}\right)_{\langle x\rangle}\right)=\chi\left(\mathbb{P} \operatorname{Ext}_{\Lambda}^{1}\left(x^{\prime}, x^{\prime \prime}\right)\right)=\operatorname{dim}_{\operatorname{Ext}_{\Lambda}^{1}}\left(x^{\prime}, x^{\prime \prime}\right),
$$

see also $\S 7.2$.

\subsection{Main result}

Theorem 1 (Multiplication formula). With the notation of $\S 1.3$, we have

$$
\chi\left(\mathbb{P} \operatorname{Ext}_{\Lambda}^{1}\left(x^{\prime}, x^{\prime \prime}\right)\right) \delta_{x^{\prime} \oplus x^{\prime \prime}}=\sum_{x \in R(\mathbf{e})}\left(\chi\left(\mathbb{P} \operatorname{Ext}_{\Lambda}^{1}\left(x^{\prime}, x^{\prime \prime}\right)_{\langle x\rangle}\right)+\chi\left(\mathbb{P} \operatorname{Ext}_{\Lambda}^{1}\left(x^{\prime \prime}, x^{\prime}\right)_{\langle x\rangle}\right)\right) \delta_{x} .
$$

This theorem is very much inspired by recent work by Caldero and Keller on cluster algebras and cluster categories of finite type [CK05].

Note that our multiplication formula is meaningless if $\operatorname{Ext}_{\Lambda}^{1}\left(x^{\prime}, x^{\prime \prime}\right)=0$ (or, equivalently, if $\left.\operatorname{Ext}_{\Lambda}^{1}\left(x^{\prime \prime}, x^{\prime}\right)=0\right)$. We proved in [GLS05a] that in all cases

$$
\delta_{x^{\prime} \oplus x^{\prime \prime}}=\delta_{x^{\prime}} \cdot \delta_{x^{\prime \prime}}
$$

For this reason we regard Theorem 1 as a multiplication formula. Using the notation from [GLS05a] 
the formula of the theorem can be rewritten as

$$
\delta_{x^{\prime}} \cdot \delta_{x^{\prime \prime}}=\frac{1}{\operatorname{dim} \operatorname{Ext}_{\Lambda}^{1}\left(x^{\prime}, x^{\prime \prime}\right)}\left(\int_{\mathbb{C}^{*}[\eta] \in \mathbb{P} \operatorname{Ext}_{\Lambda}^{1}\left(x^{\prime}, x^{\prime \prime}\right)} \delta_{\mathrm{mt}(\eta)}+\int_{\mathbb{C}^{*}[\eta] \in \mathbb{P} \operatorname{Ext}_{\Lambda}^{1}\left(x^{\prime \prime}, x^{\prime}\right)} \delta_{\operatorname{mt}(\eta)}\right) .
$$

Here, the 'integrals' are taken with respect to the 'measure' given by the Euler characteristic, $\operatorname{mt}(\eta)$ stands for the isomorphism class of the middle term of the extension $\eta$. If $[\eta] \neq 0$ we write $\mathbb{C}^{*}[\eta]$ for the corresponding element in the projective space $\mathbb{P} \operatorname{Ext}_{\Lambda}^{1}\left(x^{\prime}, x^{\prime \prime}\right)$.

The following important special case is easier to state and also easier to prove.

Theorem 2. Let $x^{\prime}$ and $x^{\prime \prime}$ be $\Lambda$-modules such that $\operatorname{dim} \operatorname{Ext}_{\Lambda}^{1}\left(x^{\prime}, x^{\prime \prime}\right)=1$, and let

$$
0 \rightarrow x^{\prime \prime} \rightarrow x \rightarrow x^{\prime} \rightarrow 0 \quad \text { and } \quad 0 \rightarrow x^{\prime} \rightarrow y \rightarrow x^{\prime \prime} \rightarrow 0
$$

be non-split short exact sequences. Then $\delta_{x^{\prime}} \cdot \delta_{x^{\prime \prime}}=\delta_{x}+\delta_{y}$.

A $\Lambda$-module $x$ is called rigid if $\operatorname{Ext}_{\Lambda}^{1}(x, x)=0$. If $x$ is rigid, it is generic and $\delta_{x}$ is a dual semicanonical basis vector. In the above theorem, if $x^{\prime}$ and $x^{\prime \prime}$ are rigid modules with $\operatorname{dim} \operatorname{Ext}_{\Lambda}^{1}\left(x^{\prime}, x^{\prime \prime}\right)=1$ one can show that $x$ and $y$ have to be rigid as well, see [GLS06]. Thus, we obtain a multiplication formula for certain dual semicanonical basis vectors. This is analyzed and interpreted in more detail in [GLS06].

\subsection{Calabi-Yau property}

For the proof of our result it is crucial that in the category of finite-dimensional (nilpotent) $\Lambda$-modules we have a functorial pairing

$$
\operatorname{Ext}_{\Lambda}^{1}(x, y) \times \operatorname{Ext}_{\Lambda}^{1}(y, x) \rightarrow \mathbb{C} .
$$

In other words, for each pair $(x, y)$ of finite-dimensional (nilpotent) $\Lambda$-modules there is an isomorphism

$$
\phi_{x, y}: \operatorname{Ext}_{\Lambda}^{1}(x, y) \rightarrow D \operatorname{Ext}_{\Lambda}^{1}(y, x)
$$

such that for any $\lambda \in \operatorname{Hom}_{\Lambda}\left(y, y^{\prime}\right),[\eta] \in \operatorname{Ext}_{\Lambda}^{1}(x, y), \rho \in \operatorname{Hom}_{\Lambda}\left(x^{\prime}, x\right)$ and $[\epsilon] \in \operatorname{Ext}_{\Lambda}^{1}\left(y^{\prime}, x^{\prime}\right)$ one has

$$
\phi_{x^{\prime}, y^{\prime}}(\lambda \circ[\eta] \circ \rho)([\epsilon])=\phi_{x, y}([\eta])(\rho \circ[\epsilon] \circ \lambda) .
$$

This is well-known to specialists. The usual argument is that this is the 'shadow' of certain 2-CalabiYau properties for preprojective algebras, see $\S 7.1$ for more details.

In particular, in the non-Dynkin case this argument relies on the fact that the preprojective algebra is a 2-Calabi-Yau algebra. Unfortunately, we could not localize a written proof for this last fact. So we include in $\S 8$ a direct proof for the required functorial isomorphism which does not depend on the type of $Q$ and does not require nilpotency for the finite-dimensional modules either.

\section{Flags and composition series induced by short exact sequences}

\subsection{Definition of $\mathcal{M}(d)$}

We repeat some notation from [GLS05a]. Recall that $I$ is the set of vertices of $Q$. We identify the elements of $I$ with the natural basis of $\mathbb{Z}^{I}$. Thus, for $\mathbf{i}=\left(i_{1}, \ldots, i_{m}\right)$ a sequence of elements of $I$, we may define $|\mathbf{i}|:=\sum_{k=1}^{m} i_{k} \in \mathbb{Z}^{I}$.

Let $\mathbf{V}$ be an $I$-graded vector space with graded dimension $|\mathbf{V}| \in \mathbb{Z}^{I}$. We denote by $\Lambda_{\mathbf{V}}$ the affine variety of $\Lambda$-module structures on $\mathbf{V}$. Clearly, if $\mathbf{V}^{\prime}$ is another graded space with $\left|\mathbf{V}^{\prime}\right|=|\mathbf{V}|$ the varieties $\Lambda_{\mathbf{V}}$ and $\Lambda_{\mathbf{V}^{\prime}}$ are isomorphic. Therefore, it is sometimes convenient to write $\Lambda_{|\mathbf{V}|}$ instead of $\Lambda_{\mathbf{V}}$ and to think of it as the variety of $\Lambda$-modules with dimension vector $\mathbf{d}=|\mathbf{V}|$. 


\section{Geiss, B. Leclerc And J. Schröer}

If $\mathbf{c}=\left(c_{1}, \ldots, c_{m}\right) \in\{0,1\}^{m}$ and $\mathbf{V}$ is an $I$-graded vector space such that $\sum_{k} c_{k} i_{k}=|\mathbf{V}|$, we define a flag in $\mathbf{V}$ of type $(\mathbf{i}, \mathbf{c})$ as a sequence

$$
\mathfrak{f}=\left(\mathbf{V}=\mathbf{V}^{0} \supseteq \mathbf{V}^{1} \supseteq \cdots \supseteq \mathbf{V}^{m}=0\right)
$$

of $I$-graded subspaces of $\mathbf{V}$ such that $\left|\mathbf{V}^{k-1} / \mathbf{V}^{k}\right|=c_{k} i_{k}$ for $k=1, \ldots, m$. We denote by $\Phi_{\mathbf{i}, \mathbf{c}}$ the variety of flags of type $(\mathbf{i}, \mathbf{c})$. When $\left(c_{1}, \ldots, c_{m}\right)=(1, \ldots, 1)$, we simply write $\Phi_{\mathbf{i}}$.

Let $x \in \Lambda_{\mathbf{V}}$. A flag $\mathfrak{f}$ is $x$-stable if $x\left(\mathbf{V}^{k}\right) \subseteq \mathbf{V}^{k}$ for all $k$. We denote by $\Phi_{\mathbf{i}, \mathbf{c}, x}$ (respectively $\Phi_{\mathbf{i}, x}$ ) the variety of $x$-stable flags of type (i, c) (respectively of type $\mathbf{i}$ ). Note that an $x$-stable flag is the same as a composition series of $x$ regarded as a $\Lambda$-module. Let $d_{\mathbf{i}, \mathbf{c}}: \Lambda_{\mathbf{V}} \rightarrow \mathbb{C}$ be the function defined by

$$
d_{\mathbf{i}, \mathbf{c}}(x)=\chi\left(\Phi_{\mathbf{i}, \mathbf{c}, x}\right) .
$$

It follows from [Lus91, $\S 2.1$ ] that $d_{\mathbf{i}, \mathbf{c}}$ is a constructible function; see also [GLS05a, $\S 5.8$ ]. If $c_{k}=1$ for all $k$, we simply write $d_{\mathbf{i}}$ instead of $d_{\mathbf{i}, \mathbf{c}}$. In general, $d_{\mathbf{i}, \mathbf{c}}=d_{\mathbf{j}}$ where $\mathbf{j}$ is the subword of $\mathbf{i}$ consisting of the letters $i_{k}$ for which $c_{k}=1$. We then define $\mathcal{M}(\mathbf{V})$ as the vector space over $\mathbb{C}$ spanned by the functions $d_{\mathbf{j}}$, where $\mathbf{j}$ runs over all words in $I$ with $|\mathbf{j}|=|\mathbf{V}|$. Again this space only depends on $\mathbf{d}=|\mathbf{V}|$, up to isomorphism, and we also denote it by $\mathcal{M}(\mathbf{d})$. This is equivalent to Lusztig's definition in [Lus91, Lus00].

\subsection{Reformulation}

In order to prove Theorem 1 and Theorem 2 it is sufficient to show that both sides of the respective equalities coincide after evaluation at an arbitrary $d_{\mathbf{i}}$, see $\S 2.1$. Since

$$
\delta_{x}\left(d_{\mathbf{i}}\right)=\chi\left(\Phi_{\mathbf{i}, x}\right)
$$

to prove Theorem 1 we have to show that

$$
\chi\left(\mathbb{P} \operatorname{Ext}_{\Lambda}^{1}\left(x^{\prime}, x^{\prime \prime}\right)\right) \cdot \chi\left(\Phi_{\mathbf{i}, x^{\prime} \oplus x^{\prime \prime}}\right)=\sum_{x \in R(\mathbf{e})}\left(\chi\left(\mathbb{P} \operatorname{Ext}_{\Lambda}^{1}\left(x^{\prime}, x^{\prime \prime}\right)_{\langle x\rangle}\right)+\chi\left(\mathbb{P} \operatorname{Ext}_{\Lambda}^{1}\left(x^{\prime \prime}, x^{\prime}\right)_{\langle x\rangle}\right)\right) \cdot \chi\left(\Phi_{\mathbf{i}, x}\right)
$$

for all sequences $\mathbf{i}$ with $|\mathbf{i}|=\mathbf{e}=\underline{\operatorname{dim}}\left(x^{\prime}\right)+\underline{\operatorname{dim}}\left(x^{\prime \prime}\right)$. Similarly, for Theorem 2 we have to show that

$$
\chi\left(\Phi_{\mathbf{i}, x^{\prime} \oplus x^{\prime \prime}}\right)=\chi\left(\Phi_{\mathbf{i}, x}\right)+\chi\left(\Phi_{\mathbf{i}, y}\right)
$$

for all sequences $\mathbf{i}$ with $|\mathbf{i}|=\underline{\operatorname{dim}}\left(x^{\prime}\right)+\underline{\operatorname{dim}}\left(x^{\prime \prime}\right)$.

2.3 Let $\mathbf{V}^{\prime}, \mathbf{V}^{\prime \prime}$, $\mathbf{V}$ be $I$-graded vector spaces such that $\left|\mathbf{V}^{\prime}\right|+\left|\mathbf{V}^{\prime \prime}\right|=|\mathbf{V}|$, and let $\mathbf{i}=\left(i_{1}, \ldots, i_{m}\right) \in$ $I^{m}$ with $\sum_{k} i_{k}=|\mathbf{V}|$. Let $x^{\prime} \in \Lambda_{\mathbf{V}^{\prime}}, x^{\prime \prime} \in \Lambda_{\mathbf{V}^{\prime \prime}}$, and $x \in \Lambda_{\mathbf{V}}$. For a short exact sequence

$$
\epsilon: 0 \rightarrow x^{\prime \prime} \stackrel{\iota}{\rightarrow} x \rightarrow x^{\prime} \rightarrow 0
$$

define a map

$$
\alpha_{\mathbf{i}, \epsilon}: \Phi_{\mathbf{i}, x} \rightarrow \bigsqcup_{\left(\mathbf{c}^{\prime}, \mathbf{c}^{\prime \prime}\right)} \Phi_{\mathbf{i}, \mathbf{c}^{\prime}, x^{\prime}} \times \Phi_{\mathbf{i}, \mathbf{c}^{\prime \prime}, x^{\prime \prime}}
$$

which maps a flag $\mathfrak{f}_{x}=\left(\mathbf{V}^{l}\right)_{0 \leqslant l \leqslant m} \in \Phi_{\mathbf{i}, x}$ of submodules of $x$ to $\left(\mathfrak{f}_{x^{\prime}}, \mathfrak{f}_{x^{\prime \prime}}\right)$ where

$$
\mathfrak{f}_{x^{\prime}}=\left(\mathbf{V}^{l} /\left(\mathbf{V}^{l} \cap \iota\left(x^{\prime \prime}\right)\right)\right)_{0 \leqslant l \leqslant m} \quad \text { and } \quad \mathfrak{f}_{x^{\prime \prime}}=\left(\mathbf{V}^{l} \cap \iota\left(x^{\prime \prime}\right)\right)_{0 \leqslant l \leqslant m} .
$$

Here we regard $\mathfrak{f}_{x^{\prime \prime}}$ as a flag in $\mathbf{V}^{\prime \prime}$ by identifying $\mathbf{V}^{\prime \prime}$ with $\iota\left(x^{\prime \prime}\right)$ and $\mathfrak{f}_{x^{\prime}}$ as a flag in $\mathbf{V}^{\prime}$ by identifying $\mathbf{V}^{\prime}$ with $\mathbf{V} / \iota\left(x^{\prime \prime}\right)$. Clearly, we have $\left(\mathfrak{f}_{x^{\prime}}, \mathfrak{f}_{x^{\prime \prime}}\right) \in \Phi_{\mathbf{i}, \mathbf{c}^{\prime}, x^{\prime}} \times \Phi_{\mathbf{i}, \mathbf{c}^{\prime \prime}, x^{\prime \prime}}$ for some sequences $\mathbf{c}^{\prime}, \mathbf{c}^{\prime \prime}$ in $\{0,1\}^{m}$ satisfying

$$
\sum_{k=1}^{m} c_{k}^{\prime} i_{k}=\left|\mathbf{V}^{\prime}\right|, \quad \sum_{k=1}^{m} c_{k}^{\prime \prime} i_{k}=\left|\mathbf{V}^{\prime \prime}\right|, \quad c_{k}^{\prime}+c_{k}^{\prime \prime}=1(1 \leqslant k \leqslant m) .
$$


Let $W_{\mathbf{V}^{\prime}, \mathbf{V}^{\prime \prime}}$ denote the set of pairs $\left(\mathbf{c}^{\prime}, \mathbf{c}^{\prime \prime}\right)$ satisfying (2.2). Define

$$
\begin{gathered}
L_{\mathbf{i}, \mathbf{c}^{\prime}, \mathbf{c}^{\prime \prime}, \epsilon}^{1}=\left(\Phi_{\mathbf{i}, \mathbf{c}^{\prime}, x^{\prime}} \times \Phi_{\mathbf{i}, \mathbf{c}^{\prime \prime}, x^{\prime \prime}}\right) \cap \operatorname{Im}\left(\alpha_{\mathbf{i}, \epsilon}\right), \\
L_{\mathbf{i}, \mathbf{c}^{\prime}, \mathbf{c}^{\prime \prime}, \epsilon}=\left(\Phi_{\mathbf{i}, \mathbf{c}^{\prime}, x^{\prime}} \times \Phi_{\mathbf{i}, \mathbf{c}^{\prime \prime}, x^{\prime \prime}}\right) \backslash L_{\mathbf{i}, \mathbf{c}^{\prime}, \mathbf{c}^{\prime \prime}, \epsilon}^{1}, \\
\Phi_{\mathbf{i}, x}\left(\mathbf{c}^{\prime}, \mathbf{c}^{\prime \prime}, \epsilon\right)=\alpha_{\mathbf{i}, \epsilon}^{-1}\left(\Phi_{\mathbf{i}, \mathbf{c}^{\prime}, x^{\prime}} \times \Phi_{\mathbf{i}, \mathbf{c}^{\prime \prime}, x^{\prime \prime}}\right) .
\end{gathered}
$$

Thus, we obtain maps

$$
\alpha_{\mathbf{i}, \epsilon}\left(\mathbf{c}^{\prime}, \mathbf{c}^{\prime \prime}\right): \Phi_{\mathbf{i}, x}\left(\mathbf{c}^{\prime}, \mathbf{c}^{\prime \prime}, \epsilon\right) \rightarrow \Phi_{\mathbf{i}, \mathbf{c}^{\prime}, x^{\prime}} \times \Phi_{\mathbf{i}, \mathbf{c}^{\prime \prime}, x^{\prime \prime}}
$$

Set $W_{\mathbf{V}^{\prime}, \mathbf{V}^{\prime \prime}, \epsilon}=\left\{\left(\mathbf{c}^{\prime}, \mathbf{c}^{\prime \prime}\right) \in W_{\mathbf{V}^{\prime}, \mathbf{V}^{\prime \prime}} \mid \Phi_{\mathbf{i}, x}\left(\mathbf{c}^{\prime}, \mathbf{c}^{\prime \prime}, \epsilon\right) \neq \emptyset\right\}$. We get a finite partition

$$
\Phi_{\mathbf{i}, x}=\bigsqcup_{\left(\mathbf{c}^{\prime}, \mathbf{c}^{\prime \prime}\right) \in W_{\mathbf{V}^{\prime}, \mathbf{V}^{\prime \prime}, \epsilon}} \Phi_{\mathbf{i}, x}\left(\mathbf{c}^{\prime}, \mathbf{c}^{\prime \prime}, \epsilon\right) .
$$

2.4 For the rest of this section let

$$
\mathfrak{f}_{x^{\prime}}=\left(x^{\prime}=x_{0}^{\prime} \supseteq x_{1}^{\prime} \supseteq \cdots \supseteq x_{m}^{\prime}=0\right) \quad \text { and } \quad \mathfrak{f}_{x^{\prime \prime}}=\left(x^{\prime \prime}=x_{0}^{\prime \prime} \supseteq x_{1}^{\prime \prime} \supseteq \cdots \supseteq x_{m}^{\prime \prime}=0\right)
$$

be flags with $\left(\mathfrak{f}_{x^{\prime}}, \mathfrak{f}_{x^{\prime \prime}}\right) \in \Phi_{\mathbf{i}, \mathbf{c}^{\prime}, x^{\prime}} \times \Phi_{\mathbf{i}, \mathbf{c}^{\prime \prime}, x^{\prime \prime}}$ for some $\left(\mathbf{c}^{\prime}, \mathbf{c}^{\prime \prime}\right) \in W_{\mathbf{V}^{\prime}, \mathbf{V}^{\prime \prime}}$ where $x^{\prime} \in \Lambda_{\mathbf{V}^{\prime}}$ and $x^{\prime \prime} \in \Lambda_{\mathbf{V}^{\prime \prime}}$.

For $1 \leqslant j \leqslant m$ let $\iota_{x^{\prime}, j}$ and $\iota_{x^{\prime \prime}, j}$ be the inclusion maps $x_{j}^{\prime} \rightarrow x_{j-1}^{\prime}$ and $x_{j}^{\prime \prime} \rightarrow x_{j-1}^{\prime \prime}$, respectively. Note that for all $j$ either $\iota_{x^{\prime}, j}$ or $\iota_{x^{\prime \prime}, j}$ is an identity map. In particular, either $x_{m-1}^{\prime}=0$ or $x_{m-1}^{\prime \prime}=0$.

All results in this section and also the definition of the maps $\beta^{\prime}$ and $\beta$ (see below) are inspired by [CK05]. The following lemma follows directly from the definitions and the considerations in $\S 7.2$.

Lemma 2.4.1. Let $\epsilon: 0 \rightarrow x^{\prime \prime} \rightarrow x \rightarrow x^{\prime} \rightarrow 0$ be a short exact sequence of $\Lambda$-modules. Then the following are equivalent:

(i) $\left(\mathfrak{f}_{x^{\prime}}, \mathfrak{f}_{x^{\prime \prime}}\right) \in L_{\mathbf{i}, \mathbf{c}^{\prime}, \mathbf{c}^{\prime \prime}, \epsilon}$;

(ii) there exists a commutative diagram with exact rows of the form

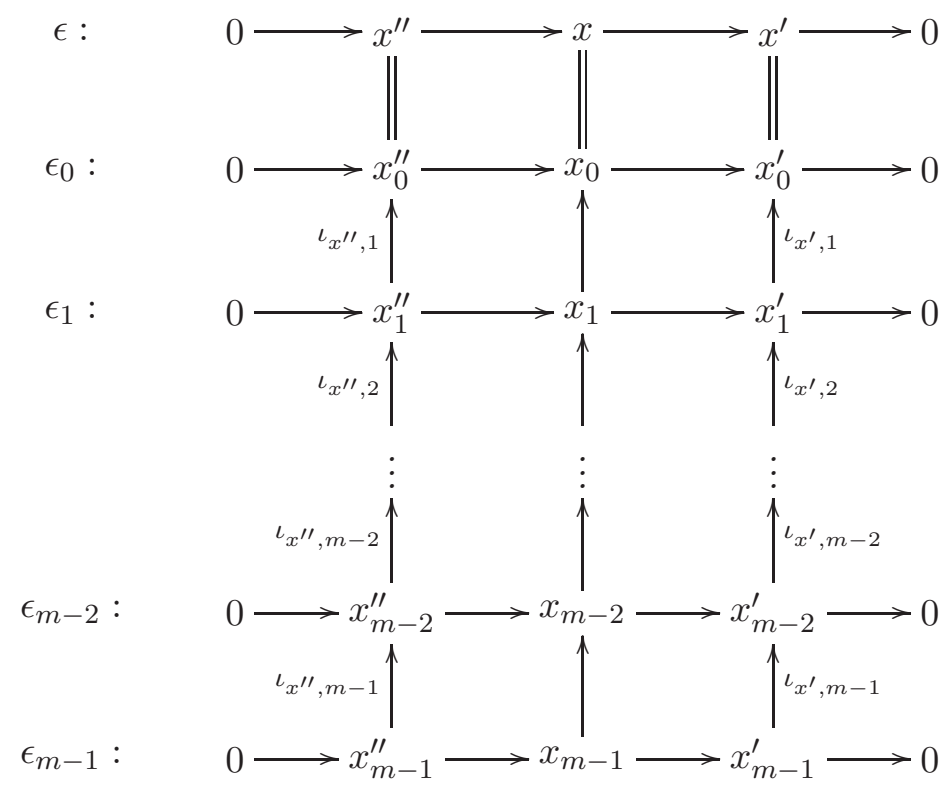

(iii) there exist elements $\left[\epsilon_{i}\right] \in \operatorname{Ext}_{\Lambda}^{1}\left(x_{i}^{\prime}, x_{i}^{\prime \prime}\right)$ for $i=0,1, \ldots, m-1$ such that $\left[\epsilon_{0}\right]=[\epsilon]$ and

$$
\left[\epsilon_{j-1}\right] \circ \iota_{x^{\prime}, j}=\iota_{x^{\prime \prime}, j} \circ\left[\epsilon_{j}\right] \quad \text { for } j=1,2, \ldots, m-1 .
$$




\section{Geiss, B. Leclerc And J. Schröer}

We define a map

$$
\begin{aligned}
\beta_{\mathbf{i}, \mathbf{c}^{\prime}, \mathbf{c}^{\prime}, \mathfrak{f}_{x^{\prime}, f_{x^{\prime \prime}}}^{\prime}}: & \bigoplus_{j=0}^{m-2} \operatorname{Ext}_{\Lambda}^{1}\left(x_{j}^{\prime}, x_{j}^{\prime \prime}\right)=: W^{\prime} \rightarrow V^{\prime}:=\bigoplus_{j=0}^{m-2} \operatorname{Ext}_{\Lambda}^{1}\left(x_{j+1}^{\prime}, x_{j}^{\prime \prime}\right) \\
& \left(\left[\epsilon_{0}\right], \ldots,\left[\epsilon_{m-2}\right]\right) \mapsto \sum_{j=0}^{m-3}\left(\left[\epsilon_{j}\right] \circ \iota_{x^{\prime}, j+1}-\iota_{x^{\prime \prime}, j+1} \circ\left[\epsilon_{j+1}\right]\right)+\left[\epsilon_{m-2}\right] \circ \iota_{x^{\prime}, m-1} .
\end{aligned}
$$

Observe that $\left[\epsilon_{j}\right] \circ \iota_{x^{\prime}, j+1}-\iota_{x^{\prime \prime}, j+1} \circ\left[\epsilon_{j+1}\right]$ is an element of $\operatorname{Ext}_{\Lambda}^{1}\left(x_{j+1}^{\prime}, x_{j}^{\prime \prime}\right)$. We denote the canonical projection map by

$$
p_{0}: \bigoplus_{j=0}^{m-2} \operatorname{Ext}_{\Lambda}^{1}\left(x_{j}^{\prime}, x_{j}^{\prime \prime}\right) \rightarrow \operatorname{Ext}_{\Lambda}^{1}\left(x_{0}^{\prime}, x_{0}^{\prime \prime}\right) .
$$

Lemma 2.4.2. For a short exact sequence $\epsilon: 0 \rightarrow x^{\prime \prime} \rightarrow x \rightarrow x^{\prime} \rightarrow 0$, the following are equivalent:

(i) $\left(\mathfrak{f}_{x^{\prime}}, \mathfrak{f}_{x^{\prime \prime}}\right) \in L_{\mathbf{i}, \mathbf{c}^{\prime}, \mathbf{c}^{\prime \prime}, \epsilon}^{1}$;

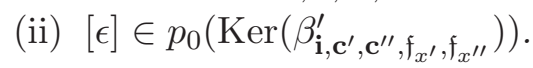

Proof. We have $[\epsilon] \in p_{0}\left(\operatorname{Ker}\left(\beta_{\mathbf{i}, \mathbf{c}^{\prime}, \mathbf{c}^{\prime \prime}, \mathfrak{f}_{x^{\prime}}, \mathfrak{f}_{x^{\prime \prime}}}^{\prime}\right)\right)$ if and only if $\beta_{\mathbf{i}, \mathbf{c}^{\prime}, \mathbf{c}^{\prime \prime}, \mathfrak{f}_{x^{\prime}}, \mathfrak{f}_{x^{\prime \prime}}}\left(\left[\epsilon_{0}\right], \ldots,\left[\epsilon_{m-2}\right]\right)=0$ for some $\left(\left[\epsilon_{0}\right], \ldots,\left[\epsilon_{m-2}\right]\right)$ with $\left[\epsilon_{0}\right]=[\epsilon]$. This is the case if and only if $\iota_{x^{\prime \prime}, j+1} \circ\left[\epsilon_{j+1}\right]=\left[\epsilon_{j}\right] \circ \iota_{x^{\prime}, j+1}$ for all $0 \leqslant j \leqslant m-3,\left[\epsilon_{m-2}\right] \circ \iota_{x^{\prime}, m-1}=0$ and $\left[\epsilon_{0}\right]=[\epsilon]$. Now the result follows from Lemma 2.4.1.

Next, we define a map

$$
\begin{aligned}
\beta_{\mathbf{i}, \mathbf{c}^{\prime \prime}, \mathbf{c}^{\prime}, \mathfrak{f}_{x^{\prime \prime}}, \mathfrak{f}_{x^{\prime}}}: & \bigoplus_{j=0}^{m-2} \operatorname{Ext}_{\Lambda}^{1}\left(x_{j}^{\prime \prime}, x_{j+1}^{\prime}\right)=: V \rightarrow W:=\bigoplus_{j=0}^{m-2} \operatorname{Ext}_{\Lambda}^{1}\left(x_{j}^{\prime \prime}, x_{j}^{\prime}\right) \\
& \left(\left[\eta_{0}\right], \ldots,\left[\eta_{m-2}\right]\right) \mapsto \iota_{x^{\prime}, 1} \circ\left[\eta_{0}\right]+\sum_{j=1}^{m-2}\left(\iota_{x^{\prime}, j+1} \circ\left[\eta_{j}\right]-\left[\eta_{j-1}\right] \circ \iota_{x^{\prime \prime}, j}\right) .
\end{aligned}
$$

Note that $\iota_{x^{\prime}, j+1} \circ\left[\eta_{j}\right]-\left[\eta_{j-1}\right] \circ \iota_{x^{\prime \prime}, j}$ is an element of $\operatorname{Ext}_{\Lambda}^{1}\left(x_{j}^{\prime \prime}, x_{j}^{\prime}\right)$.

Lemma 2.4.3. For a short exact sequence $\eta: 0 \rightarrow x^{\prime} \rightarrow y \rightarrow x^{\prime \prime} \rightarrow 0$ the following are equivalent:

(i) $\left(\mathfrak{f}_{x^{\prime \prime}}, \mathfrak{f}_{x^{\prime}}\right) \in L_{\mathbf{i}, \mathbf{c}^{\prime \prime}, \mathbf{c}^{\prime}, \eta}^{1}$;

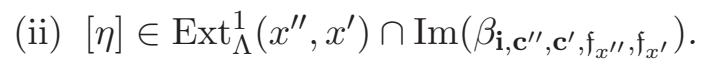

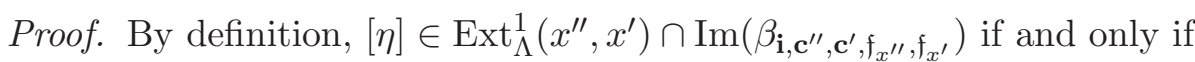

$$
([\eta], 0, \ldots, 0)=\beta_{\mathbf{i}, \mathbf{c}^{\prime \prime}, \mathbf{c}^{\prime}, f_{x^{\prime \prime}}, f_{x^{\prime}}}\left(\left[\eta_{0}\right], \ldots,\left[\eta_{m-2}\right]\right),
$$

in other words if and only if there exists $\left(\left[\eta_{0}\right], \ldots,\left[\eta_{m-2}\right]\right)$ such that $\iota_{x^{\prime}, 1} \circ\left[\eta_{0}\right]=[\eta]$ and $\left[\eta_{j-1}\right] \circ \iota_{x^{\prime \prime}, j}=$ $\iota_{x^{\prime}, j+1} \circ\left[\eta_{j}\right]$ for all $1 \leqslant j \leqslant m-2$.

On the other hand, by Lemma $2.4 .1\left(\mathfrak{f}_{x^{\prime \prime}}, \mathfrak{f}_{x^{\prime}}\right) \in L_{\mathbf{i}, \mathbf{c}^{\prime \prime}, \mathbf{c}^{\prime}, \eta}^{1}$ if and only if for $0 \leqslant i \leqslant m-1$ there exist $\left[\eta_{i}^{-}\right] \in \operatorname{Ext}_{\Lambda}^{1}\left(x_{i}^{\prime \prime}, x_{i}^{\prime}\right)$ such that $\left[\eta_{0}^{-}\right]=[\eta]$ and $\left[\eta_{i-1}^{-}\right] \circ \iota_{x^{\prime \prime}, i}=\iota_{x^{\prime}, i} \circ\left[\eta_{i}^{-}\right]$for $1 \leqslant i \leqslant m-1$.

Taking into account that for each $i$ either $\iota_{x^{\prime}, i}$ or $\iota_{x^{\prime \prime}, i}$ is an identity, it is now easy to see that the two conditions are equivalent.

2.5 As before, let $x^{\prime} \in \Lambda_{\mathbf{V}^{\prime}}, x^{\prime \prime} \in \Lambda_{\mathbf{V}^{\prime \prime}}$, and let

$$
\mathfrak{f}_{x^{\prime}}=\left(x^{\prime}=x_{0}^{\prime} \supseteq x_{1}^{\prime} \supseteq \cdots \supseteq x_{m}^{\prime}=0\right) \quad \text { and } \quad \mathfrak{f}_{x^{\prime \prime}}=\left(x^{\prime \prime}=x_{0}^{\prime \prime} \supseteq x_{1}^{\prime \prime} \supseteq \cdots \supseteq x_{m}^{\prime \prime}=0\right)
$$

be flags with $\left(\mathfrak{f}_{x^{\prime}}, \mathfrak{f}_{x^{\prime \prime}}\right) \in \Phi_{\mathbf{i}, \mathbf{c}^{\prime}, x^{\prime}} \times \Phi_{\mathbf{i}, \mathbf{c}^{\prime \prime}, x^{\prime \prime}}$ for some $\left(\mathbf{c}^{\prime}, \mathbf{c}^{\prime \prime}\right) \in W_{\mathbf{V}^{\prime}, \mathbf{V}^{\prime \prime}}$. 


\section{SEMICANONICAL BASES AND PREPROJECTIVE ALGEBRAS II}

Due to the Calabi-Yau property of $\Lambda$, see $\S 1.5$, we have non-degenerate natural pairings

$$
\begin{aligned}
& \phi_{V}: V \times V^{\prime} \rightarrow \mathbb{C}, \quad\left(\left(\left[\eta_{0}\right], \ldots,\left[\eta_{m-2}\right]\right),\left(\left[\delta_{0}\right], \ldots,\left[\delta_{m-2}\right]\right)\right) \mapsto \sum_{j=0}^{m-2} \phi_{x_{j}^{\prime \prime} x_{j+1}^{\prime}}\left(\left[\eta_{j}\right]\right)\left(\left[\delta_{j}\right]\right) \\
& \phi_{W}: W \times W^{\prime} \rightarrow \mathbb{C}, \quad\left(\left(\left[\psi_{0}\right], \ldots,\left[\psi_{m-2}\right]\right),\left(\left[\epsilon_{0}\right], \ldots,\left[\epsilon_{m-2}\right]\right)\right) \mapsto \sum_{j=0}^{m-2} \phi_{x_{j}^{\prime \prime}, x_{j}^{\prime}}\left(\left[\psi_{j}\right]\right)\left(\left[\epsilon_{j}\right]\right)
\end{aligned}
$$

for the spaces $\left(V, V^{\prime}\right)$ and $\left(W, W^{\prime}\right)$ defined in (2.3) and (2.4).

Lemma 2.5.1. For all $\left(\left[\eta_{0}\right], \ldots,\left[\eta_{m-2}\right]\right) \in V$ and $\left(\left[\epsilon_{0}\right], \ldots,\left[\epsilon_{m-2}\right]\right) \in W^{\prime}$ we have

$$
\phi_{V}\left(\left(\left[\eta_{0}\right], \ldots,\left[\eta_{m-2}\right]\right), \beta^{\prime}\left(\left(\left[\epsilon_{0}\right], \ldots,\left[\epsilon_{m-2}\right]\right)\right)\right)=\phi_{W}\left(\beta\left(\left(\left[\eta_{0}\right], \ldots,\left[\eta_{m-2}\right]\right)\right),\left(\left[\epsilon_{0}\right], \ldots,\left[\epsilon_{m-2}\right]\right)\right) .
$$

Proof. By definition, the left-hand side of the equation is

$$
\sum_{j=0}^{m-3} \phi_{x_{j}^{\prime \prime}, x_{j+1}^{\prime}}\left(\left[\eta_{j}\right]\right)\left(\left[\epsilon_{j}\right] \circ \iota_{x^{\prime}, j+1}-\iota_{x^{\prime \prime}, j+1} \circ\left[\epsilon_{j+1}\right]\right)+\phi_{x_{m-2}^{\prime \prime}, x_{m-1}^{\prime}}\left(\left[\eta_{m-1}\right]\right)\left(\left[\epsilon_{m-2}\right] \circ \iota_{x_{m-1}^{\prime \prime}}\right),
$$

using (1.1), this is equal to

$$
\phi_{x_{0}^{\prime \prime}, x_{0}^{\prime}}\left(\iota_{x^{\prime}, 1} \circ\left[\eta_{0}\right]\right)\left(\left[\epsilon_{0}\right]\right)+\sum_{j=1}^{m-2} \phi_{x_{j}^{\prime \prime}, x_{j}^{\prime}}\left(\iota_{x^{\prime}, j+1} \circ\left[\eta_{j}\right]-\left[\eta_{j-1}\right] \circ \iota_{x^{\prime \prime}, j}\right)\left(\left[\epsilon_{j}\right]\right),
$$

which is by definition the right-hand side of our claim.

Proposition 2.5.2. For $[\epsilon] \in \operatorname{Ext}_{\Lambda}^{1}\left(x^{\prime}, x^{\prime \prime}\right)$ the following are equivalent:

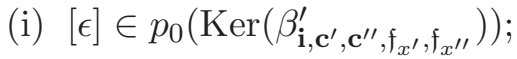

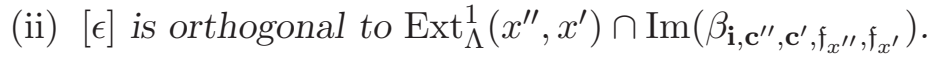

Proof. By Lemmas 7.3.1 and 2.5.1 we have

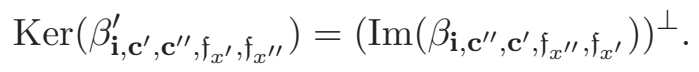

Putting $W_{0}^{\prime}=\operatorname{Ext}_{\Lambda}^{1}\left(x_{0}^{\prime}, x_{0}^{\prime \prime}\right)=\operatorname{Ext}_{\Lambda}^{1}\left(x^{\prime}, x^{\prime \prime}\right)$ and $W_{0}=\operatorname{Ext}_{\Lambda}^{1}\left(x_{0}^{\prime \prime}, x_{0}^{\prime}\right)=\operatorname{Ext}_{\Lambda}^{1}\left(x^{\prime \prime}, x^{\prime}\right)$, it follows that

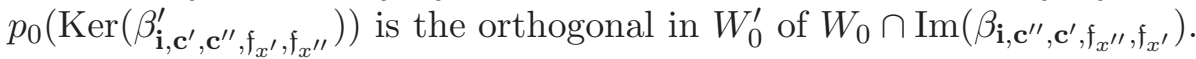

Corollary 2.5.3. Assume that $\operatorname{dim}_{\operatorname{Ext}_{\Lambda}^{1}}\left(x^{\prime}, x^{\prime \prime}\right)=1$, and let

$$
\epsilon: 0 \rightarrow x^{\prime \prime} \rightarrow x \rightarrow x^{\prime} \rightarrow 0 \text { and } \eta: 0 \rightarrow x^{\prime} \rightarrow y \rightarrow x^{\prime \prime} \rightarrow 0
$$

be non-split short exact sequences. Then the following are equivalent:

(i) $\left(\mathfrak{f}_{x^{\prime}}, \mathfrak{f}_{x^{\prime \prime}}\right) \in L_{\mathbf{i}, \mathbf{c}^{\prime}, \mathbf{c}^{\prime \prime}, \epsilon}^{1}$;

(ii) $\left(\mathfrak{f}_{x^{\prime \prime}}, \mathfrak{f}_{x^{\prime}}\right) \in L_{\mathbf{i}, \mathbf{c}^{\prime \prime}, \mathbf{c}^{\prime}, \eta}^{2}$.

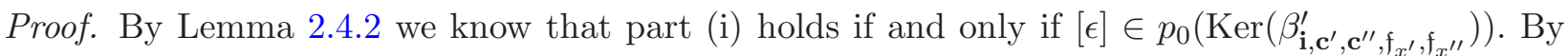

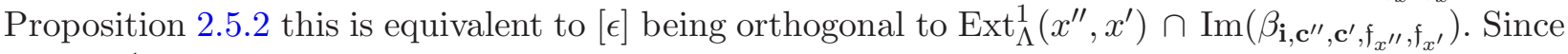
$\operatorname{dim} \operatorname{Ext}_{\Lambda}^{1}\left(x^{\prime \prime}, x^{\prime}\right)=1$ and since $\epsilon$ is non-split, this is the case if and only if

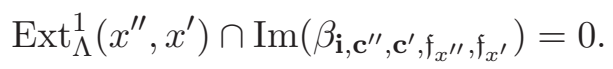

By Lemma 2.4.3 and the fact that $\eta$ is non-split this happens if and only if $\left(\mathfrak{f}_{x^{\prime \prime}}, \mathfrak{f}_{x^{\prime}}\right) \in L_{\mathbf{i}, \mathbf{c}^{\prime \prime}, \mathbf{c}^{\prime}, \eta}^{2}$.

Let us stress that Proposition 2.5.2 is very similar to [CK05, Proposition 4]. We just had to adapt Caldero and Keller's result to our setting of preprojective algebras. Our proof then follows the main line of their proof. In fact, our situation turns out to be easier to handle, because we work with modules and not with objects in cluster categories. 


\section{Geiss, B. Leclerc And J. Schröer}

\section{Euler characteristics of flag varieties}

This section is inspired by the proof of [Lus91, Lemma 4.4].

3.1 Let $\mathbf{V}, \mathbf{W}$ and $\mathbf{T}$ be $I$-graded vector spaces with $\mathbf{V}=\mathbf{T} \oplus \mathbf{W}$. We identify $\mathbf{V} / \mathbf{W}$ with $\mathbf{T}$. We write $m=\operatorname{dim} \mathbf{V}$. Let $\Phi(\mathbf{V})$ denote the variety of complete $I$-graded flags in $\mathbf{V}$. Let

$$
\pi: \Phi(\mathbf{V}) \rightarrow \Phi_{m}(\mathbf{T}) \times \Phi_{m}(\mathbf{W})
$$

be the morphism which maps a flag $\left(\mathbf{V}^{l}\right)_{0 \leqslant l \leqslant m}$ to the pair of flags $\left(\left(\mathbf{T}^{l}\right)_{0 \leqslant l \leqslant m},\left(\mathbf{W}^{l}\right)_{0 \leqslant l \leqslant m}\right)$, defined by $\mathbf{W}^{l}=\mathbf{W} \cap \mathbf{V}^{l}$ and $\mathbf{T}^{l}=p_{1}\left(\mathbf{V}^{l}\right)$. Here

$$
p_{1}: \mathbf{V}=\mathbf{T} \oplus \mathbf{W} \rightarrow \mathbf{T}
$$

is just the projection onto $\mathbf{T}$, and $\Phi_{m}(\mathbf{T})$ and $\Phi_{m}(\mathbf{W})$ denote the varieties of $m$-step $I$-graded flags in $\mathbf{W}$ and $\mathbf{T}$, respectively. Note that $\mathbf{T}^{j} / \mathbf{T}^{j+1}$ and $\mathbf{W}^{j} / \mathbf{W}^{j+1}$ have dimension 0 or 1 for all $j$. We want to study the fibers of the morphism $\pi$.

Let $\left(\mathfrak{f}^{\prime}=\left(\mathbf{T}^{l}\right), \mathfrak{f}^{\prime \prime}=\left(\mathbf{W}^{l}\right)\right) \in \operatorname{Im}(\pi)$. For an $I$-graded linear map $z: \mathbf{T} \rightarrow \mathbf{W}$ we define a flag

$$
\mathfrak{f}_{z}=\mathfrak{f}_{z, \mathfrak{f}^{\prime}, \mathfrak{f}^{\prime \prime}}=\left(\mathbf{V}_{z}^{l}\right)_{0 \leqslant l \leqslant m} \in \Phi(\mathbf{V})
$$

by

$$
\mathbf{V}_{z}^{l}=\left(0, \mathbf{W}^{l}\right)+\left\{(t, z(t)) \mid t \in \mathbf{T}^{l}\right\} \subseteq \mathbf{T}^{l} \oplus \mathbf{W} .
$$

Clearly, we have $\mathfrak{f}_{z} \in \pi^{-1}\left(\mathfrak{f}^{\prime}, \mathfrak{f}^{\prime \prime}\right)$.

We say that two $I$-graded linear maps $f, g: \mathbf{T} \rightarrow \mathbf{W}$ are equivalent if $\mathbf{V}_{f}^{l}=\mathbf{V}_{g}^{l}$ for all $l$. In this case we write $f \sim_{\mathfrak{f}^{\prime}, f^{\prime \prime}} g$ or just $f \sim g$. In other words, $f$ and $g$ are equivalent if and only if $(f-g)\left(\mathbf{T}^{l}\right) \subseteq \mathbf{W}^{l}$ for all $l$.

The next lemma shows that every flag in $\pi^{-1}\left(\mathfrak{f}^{\prime}, \mathfrak{f}^{\prime \prime}\right)$ is of the form $\mathfrak{f}_{z}$ for some $z$.

Lemma 3.1.1. If $\pi(\mathfrak{f})=\left(\mathfrak{f}^{\prime}, \mathfrak{f}^{\prime \prime}\right)$, then there exists an I-graded linear map $z: \mathbf{T} \rightarrow \mathbf{W}$ such that $\mathfrak{f}=\mathfrak{f}_{z, \mathfrak{f}^{\prime}, \mathfrak{f}^{\prime \prime}}$.

Proof. Choose a decomposition for the two graded vector spaces

$$
\mathbf{T}=\bigoplus_{i=0}^{m} \mathbf{T}(i) \text { and } \mathbf{W}=\bigoplus_{i=0}^{m} \mathbf{W}(i)
$$

such that

$$
\mathbf{T}^{l}=\bigoplus_{i=l}^{m} \mathbf{T}(i) \quad \text { and } \quad \mathbf{W}^{l}=\bigoplus_{i=l}^{m} \mathbf{W}(i)
$$

for all $l$. Put

$$
\mathbf{W}_{c}^{l}:=\bigoplus_{i=0}^{l-1} \mathbf{W}(i)
$$

and take a flag $\mathfrak{f}=\left(\mathbf{V}^{l}\right)_{0 \leqslant l \leqslant m}$ such that $\pi(\mathfrak{f})=\left(\mathfrak{f}^{\prime}, \mathfrak{f}^{\prime \prime}\right)$. Since we have a short exact sequence

$$
0 \rightarrow \mathbf{W}^{l} \rightarrow \mathbf{V}^{l} \rightarrow \mathbf{T}^{l} \rightarrow 0,
$$

there exists a unique $I$-graded linear map $w^{l}: \mathbf{T}^{l} \rightarrow \mathbf{W}_{c}^{l}$ such that

$$
\mathbf{V}^{l}=\left(0, \mathbf{W}^{l}\right)+\left\{\left(t, w^{l}(t)\right) \mid t \in \mathbf{T}^{l}\right\} .
$$

The conditions $\mathbf{V}^{l} \supset \mathbf{V}^{l+1}$ and $\mathbf{W}_{c}^{l} \subseteq \mathbf{W}_{c}^{l+1}$ imply

$$
w^{l+1}(t)-w^{l}(t) \in \mathbf{W}_{c}^{l+1} \cap \mathbf{W}^{l}=\mathbf{W}(l)
$$




\section{SEMICANONICAL BASES AND PREPROJECTIVE ALGEBRAS II}

for all $t \in \mathbf{T}^{l+1}$. Hence, for $t \in \mathbf{T}^{l+1}$ we have

$$
\left(t, w^{l+1}(t)\right)-\left(t, w^{l}(t)\right)=\left(0, w^{l+1}(t)-w^{l}(t)\right) \in \mathbf{W}^{l} \subseteq \mathbf{V}^{l} .
$$

Now, define $z: \mathbf{T} \rightarrow \mathbf{W}$ on the summands of $\mathbf{T}$ by $z(t)=w^{i}(t)$ for $t \in \mathbf{T}(i)$.

Thus, the fiber $\pi^{-1}\left(\mathfrak{f}^{\prime}, \mathfrak{f}^{\prime \prime}\right)$ can be identified with the vector space

$$
\operatorname{Hom}(\mathbf{T}, \mathbf{W}) / \sim_{\mathfrak{f}^{\prime}, f^{\prime \prime}}
$$

of $I$-graded linear maps $\mathbf{T} \rightarrow \mathbf{W}$ up to equivalence.

3.2 Let $\Gamma=\mathbb{C} Q^{\prime} / J$ be an algebra where $Q^{\prime}$ is a finite quiver with set of vertices $I$ and $J$ is an ideal in the path algebra $\mathbb{C} Q^{\prime}$. For a finite-dimensional $I$-graded vector space $\mathbf{U}$ let $\Gamma_{\mathbf{U}}$ be the affine variety of $\Gamma$-module structures on $\mathbf{U}$. Fix a short exact sequence

$$
\epsilon: 0 \rightarrow x^{\prime \prime} \rightarrow x \rightarrow x^{\prime} \rightarrow 0
$$

of $\Gamma$-modules with $x \in \Gamma_{\mathbf{V}}, x^{\prime} \in \Gamma_{\mathbf{T}}$ and $x^{\prime \prime} \in \Gamma_{\mathbf{W}}$. To an $I$-graded linear map $z: \mathbf{T} \rightarrow \mathbf{W}$ we associate linear maps $z^{l}: \mathbf{T}^{l} \rightarrow \mathbf{W} / \mathbf{W}^{l}$ defined by $z^{l}(t)=z(t)+\mathbf{W}^{l}$. For a vertex $i \in I$ let $z_{i}: \mathbf{T}_{i} \rightarrow \mathbf{W}_{i}$ be the degree $i$ part of $z$. The next lemma follows from the definitions.

LEMma 3.2.1. If $x=x^{\prime} \oplus x^{\prime \prime}$ and if $\mathfrak{f}^{\prime}$ and $\mathfrak{f}^{\prime \prime}$ are flags of submodules of $x^{\prime}$ and $x^{\prime \prime}$, respectively, then $\mathfrak{f}_{z, f^{\prime}, f^{\prime \prime}}$ is a flag of submodules of $x^{\prime} \oplus x^{\prime \prime}$ if and only if the map $z^{l}$ is a module homomorphism for all $l$.

We now deal with the case when the short exact sequence $\epsilon$ does not split. A module $m \in \Gamma_{\mathbf{V}}$ can be interpreted as a tuple $m=(m(a))_{a}$ where for each arrow $a \in Q_{1}^{\prime}$ we have a linear map $m(a): \mathbf{V}_{s(a)} \rightarrow \mathbf{V}_{e(a)}$ where $s(a)$ and $e(a)$ denote the start and end vertex of the arrow $a$. Thus, given our short exact sequence

$$
\epsilon: 0 \rightarrow x^{\prime \prime} \rightarrow x \rightarrow x^{\prime} \rightarrow 0,
$$

we can assume without loss of generality that the linear maps $x(a)$ are of the form

$$
x(a)=\left(\begin{array}{cc}
x^{\prime}(a) & 0 \\
y(a) & x^{\prime \prime}(a)
\end{array}\right),
$$

where $y(a): \mathbf{T}_{s(a)} \rightarrow \mathbf{W}_{e(a)}$ are certain linear maps. The proof of the following statement is again straightforward, compare also the proof of [Lus91, Lemma 4.4].

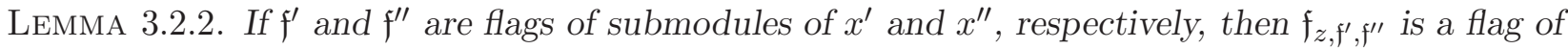
submodules of $x$ if and only if

$$
\left(x^{\prime \prime}(a) z_{s(a)}-z_{e(a)} x^{\prime}(a)-y(a)\right)\left(\mathbf{T}_{s(a)}^{l}\right) \subseteq \mathbf{W}_{e(a)}^{l}
$$

for all $0 \leqslant l \leqslant m-1$ and $a \in Q_{1}^{\prime}$.

3.3 Now we apply the above results to the case of the preprojective algebra $\Lambda$.

Lemma 3.3.1. For a short exact sequence $\epsilon: 0 \rightarrow x^{\prime \prime} \rightarrow x \rightarrow x^{\prime} \rightarrow 0$ and $\left(\mathbf{c}^{\prime}, \mathbf{c}^{\prime \prime}\right) \in W_{\mathbf{V}^{\prime}, \mathbf{V}^{\prime \prime}, \epsilon}$ the fibers of the morphism

$$
\alpha_{\mathbf{i}, \epsilon}\left(\mathbf{c}^{\prime}, \mathbf{c}^{\prime \prime}\right): \Phi_{\mathbf{i}, x}\left(\mathbf{c}^{\prime}, \mathbf{c}^{\prime \prime}, \epsilon\right) \rightarrow \Phi_{\mathbf{i}, \mathbf{c}^{\prime}, x^{\prime}} \times \Phi_{\mathbf{i}, \mathbf{c}^{\prime \prime}, x^{\prime \prime}}
$$

are isomorphic to affine spaces, moreover $\operatorname{Im}\left(\alpha_{\mathbf{i}, \epsilon}\left(\mathbf{c}^{\prime}, \mathbf{c}^{\prime \prime}\right)\right)=L_{\mathbf{i}, \mathbf{c}^{\prime}, \mathbf{c}^{\prime \prime}, \epsilon}^{1}$.

Proof. The condition in Lemmas 3.2.1 and 3.2.2 are both linear. The last equation follows from the definitions, see $\S 2.3$. 


\section{Geiss, B. Leclerc And J. Schröer}

Corollary 3.3.2. For a short exact sequence $\epsilon: 0 \rightarrow x^{\prime \prime} \rightarrow x \rightarrow x^{\prime} \rightarrow 0$ and $\left(\mathbf{c}^{\prime}, \mathbf{c}^{\prime \prime}\right) \in W_{\mathbf{V}^{\prime}, \mathbf{V}^{\prime \prime}}$ we have

$$
\chi\left(\Phi_{\mathbf{i}, x}\left(\mathbf{c}^{\prime}, \mathbf{c}^{\prime \prime}, \epsilon\right)\right)=\chi\left(L_{\mathbf{i}, \mathbf{c}^{\prime}, \mathbf{c}^{\prime \prime}, \epsilon}^{1}\right) .
$$

Proof. This follows from Lemma 3.3.1 and Proposition 7.4.1.

Corollary 3.3.3. If $x=x^{\prime} \oplus x^{\prime \prime}$, then for all short exact sequences $\epsilon: 0 \rightarrow x^{\prime \prime} \rightarrow x \rightarrow x^{\prime} \rightarrow 0$ and $\left(\mathbf{c}^{\prime}, \mathbf{c}^{\prime \prime}\right) \in W_{\mathbf{V}^{\prime}, \mathbf{V}^{\prime \prime}}$ we have

$$
\chi\left(\Phi_{\mathbf{i}, x}\left(\mathbf{c}^{\prime}, \mathbf{c}^{\prime \prime}, \epsilon\right)\right)=\chi\left(\Phi_{\mathbf{i}, \mathbf{c}^{\prime}, x^{\prime}} \times \Phi_{\mathbf{i}, \mathbf{c}^{\prime \prime}, x^{\prime \prime}}\right)=\chi\left(\Phi_{\mathbf{i}, \mathbf{c}^{\prime}, x^{\prime}}\right) \cdot \chi\left(\Phi_{\mathbf{i}, \mathbf{c}^{\prime \prime}, x^{\prime \prime}}\right) .
$$

Proof. If $x=x^{\prime} \oplus x^{\prime \prime}$ and $\left(\mathbf{c}^{\prime}, \mathbf{c}^{\prime \prime}\right) \in W_{\mathbf{V}^{\prime}, \mathbf{V}^{\prime \prime}}$, then $L_{\mathbf{i}, \mathbf{c}^{\prime}, \mathbf{c}^{\prime \prime}, \epsilon}^{1}=\Phi_{\mathbf{i}, \mathbf{c}^{\prime}, x^{\prime}} \times \Phi_{\mathbf{i}, \mathbf{c}^{\prime \prime}, x^{\prime \prime}}$ for all $\epsilon$, see [GLS05a].

In [GLS05a] we claimed that for $\left(\mathbf{c}^{\prime}, \mathbf{c}^{\prime \prime}\right) \in W_{\mathbf{V}^{\prime}, \mathbf{V}^{\prime \prime}, \epsilon}$ the map $\Phi_{\mathbf{i}, x^{\prime} \oplus x^{\prime \prime}}\left(\mathbf{c}^{\prime}, \mathbf{c}^{\prime \prime}, \epsilon\right) \rightarrow \Phi_{\mathbf{i}, \mathbf{c}^{\prime}, x^{\prime}} \times \Phi_{\mathbf{i}, \mathbf{c}^{\prime \prime}, x^{\prime \prime}}$ is a vector bundle, referring to [Lus91, Lemma 4.4]. What we had in mind was an argument as above. However, this only proves that the fibers of this map are affine spaces. Nevertheless, for the Euler characteristic calculation needed in [GLS05a] this is enough because of Proposition 7.4.1.

\section{Proof of Theorem 2}

Assume $\operatorname{dim} \operatorname{Ext}_{\Lambda}^{1}\left(x^{\prime}, x^{\prime \prime}\right)=1$, and let

$$
\epsilon: 0 \rightarrow x^{\prime \prime} \rightarrow x \rightarrow x^{\prime} \rightarrow 0 \text { and } \eta: 0 \rightarrow x^{\prime} \rightarrow y \rightarrow x^{\prime \prime} \rightarrow 0
$$

be non-split short exact sequences. We obtain the following diagram of maps.

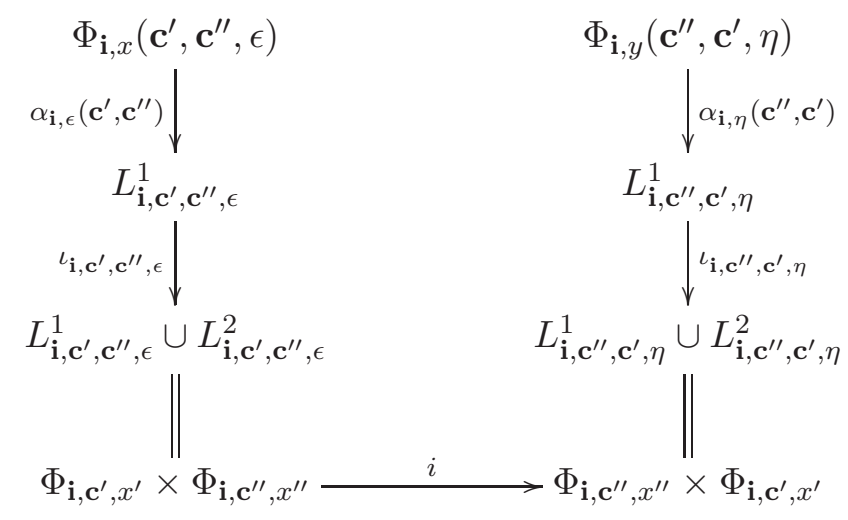

Here, the map $i$ is the isomorphism which maps $\left(\mathfrak{f}_{x^{\prime}}, \mathfrak{f}_{x^{\prime \prime}}\right)$ to $\left(\mathfrak{f}_{x^{\prime \prime}}, \mathfrak{f}_{x^{\prime}}\right)$, and $\iota_{\mathbf{i}, \mathbf{c}^{\prime}, \mathbf{c}^{\prime \prime}, \epsilon}$ and $\iota_{\mathbf{i}, \mathbf{c}^{\prime \prime}, \mathbf{c}^{\prime}, \eta}$ are the natural inclusion maps. By Corollary 3.3.2,

$$
\chi\left(\Phi_{\mathbf{i}, x}\left(\mathbf{c}^{\prime}, \mathbf{c}^{\prime \prime}, \epsilon\right)\right)=\chi\left(L_{\mathbf{i}, \mathbf{c}^{\prime}, \mathbf{c}^{\prime \prime}, \epsilon}^{1}\right) \quad \text { and } \quad \chi\left(\Phi_{\mathbf{i}, y}\left(\mathbf{c}^{\prime \prime}, \mathbf{c}^{\prime}, \eta\right)\right)=\chi\left(L_{\mathbf{i}, \mathbf{c}^{\prime \prime}, \mathbf{c}^{\prime}, \eta}^{1}\right) .
$$

We know from Corollary 3.3.3 that

$$
\chi\left(\Phi_{\mathbf{i}, x^{\prime} \oplus x^{\prime \prime}}\left(\mathbf{c}^{\prime}, \mathbf{c}^{\prime \prime}, \theta\right)\right)=\chi\left(\Phi_{\mathbf{i}, \mathbf{c}^{\prime}, x^{\prime}} \times \Phi_{\mathbf{i}, \mathbf{c}^{\prime \prime}, x^{\prime \prime}}\right)=\chi\left(L_{\mathbf{i}, \mathbf{c}^{\prime}, \mathbf{c}^{\prime \prime}, \epsilon}^{1}\right)+\chi\left(L_{\mathbf{i}, \mathbf{c}^{\prime}, \mathbf{c}^{\prime \prime}, \epsilon}^{2}\right),
$$

where $\theta$ is any (split) short exact sequence of the form $0 \rightarrow x^{\prime \prime} \rightarrow x^{\prime \prime} \oplus x^{\prime} \rightarrow x^{\prime} \rightarrow 0$. By Corollary 2.5.3 the isomorphism $i$ induces isomorphisms

$$
i_{1,2}: L_{\mathbf{i}, \mathbf{c}^{\prime}, \mathbf{c}^{\prime \prime}, \epsilon}^{1} \rightarrow L_{\mathbf{i}, \mathbf{c}^{\prime \prime}, \mathbf{c}^{\prime}, \eta}^{2} \quad \text { and } \quad i_{2,1}: L_{\mathbf{i}, \mathbf{c}^{\prime}, \mathbf{c}^{\prime \prime}, \epsilon}^{2} \rightarrow L_{\mathbf{i}, \mathbf{c}^{\prime \prime}, \mathbf{c}^{\prime}, \eta}^{1}
$$


SEMicANonical BASES AND PREPROJECTIVE ALGEBRAS II

which implies $\chi\left(L_{\mathbf{i}, \mathbf{c}^{\prime}, \mathbf{c}^{\prime \prime}, \epsilon}^{2}\right)=\chi\left(L_{\mathbf{i}, \mathbf{c}^{\prime \prime}, \mathbf{c}^{\prime}, \eta}^{1}\right)$. Combining these facts we get

$$
\begin{aligned}
\chi\left(\Phi_{\mathbf{i}, x^{\prime} \oplus x^{\prime \prime}}\right) & =\sum_{\left(\mathbf{c}^{\prime}, \mathbf{c}^{\prime \prime}\right) \in W_{\mathbf{V}^{\prime}, \mathbf{V}^{\prime \prime}}} \chi\left(\Phi_{\mathbf{i}, x^{\prime} \oplus x^{\prime \prime}}\left(\mathbf{c}^{\prime}, \mathbf{c}^{\prime \prime}, \theta\right)\right) \\
& =\sum_{\left(\mathbf{c}^{\prime}, \mathbf{c}^{\prime \prime}\right) \in W_{\mathbf{V}^{\prime}, \mathbf{V}^{\prime \prime}}} \chi\left(L_{\mathbf{i}, \mathbf{c}^{\prime}, \mathbf{c}^{\prime \prime}, \epsilon}^{1}\right)+\sum_{\left(\mathbf{c}^{\prime}, \mathbf{c}^{\prime \prime}\right) \in W_{\mathbf{V}^{\prime}, \mathbf{V}^{\prime \prime}}} \chi\left(L_{\mathbf{i}, \mathbf{c}^{\prime}, \mathbf{c}^{\prime \prime}, \epsilon}^{2}\right) \\
& =\sum_{\left(\mathbf{c}^{\prime}, \mathbf{c}^{\prime \prime}\right) \in W_{\mathbf{V}^{\prime}, \mathbf{V}^{\prime \prime}}} \chi\left(L_{\mathbf{i}, \mathbf{c}^{\prime}, \mathbf{c}^{\prime \prime}, \epsilon}^{1}\right)+\sum_{\left(\mathbf{c}^{\prime}, \mathbf{c}^{\prime \prime}\right) \in W_{\mathbf{V}^{\prime}, \mathbf{V}^{\prime \prime}}} \chi\left(L_{\mathbf{i}, \mathbf{c}^{\prime \prime}, \mathbf{c}^{\prime}, \eta}^{1}\right) \\
& =\sum_{\left(\mathbf{c}^{\prime}, \mathbf{c}^{\prime \prime}\right) \in W_{\mathbf{V}^{\prime}, \mathbf{V}^{\prime \prime}}} \chi\left(\Phi_{\mathbf{i}, x}\left(\mathbf{c}^{\prime}, \mathbf{c}^{\prime \prime}, \epsilon\right)\right)+\sum_{\left(\mathbf{c}^{\prime}, \mathbf{c}^{\prime \prime}\right) \in W_{\mathbf{V}^{\prime}, \mathbf{V}^{\prime \prime}}} \chi\left(\Phi_{\mathbf{i}, y}\left(\mathbf{c}^{\prime \prime}, \mathbf{c}^{\prime}, \eta\right)\right) \\
& =\chi\left(\Phi_{\mathbf{i}, x}\right)+\chi\left(\Phi_{\mathbf{i}, y}\right) .
\end{aligned}
$$

By the considerations in $\S 2.2$ this finishes the proof of Theorem 2 .

\section{The general case}

\subsection{Derivations}

For $\Lambda$-modules $x^{\prime}$ and $x^{\prime \prime}$ let $D_{\Lambda}\left(x^{\prime}, x^{\prime \prime}\right)$ be the vector space of all tuples $d=(d(b))_{b \in \bar{Q}_{1}}$ of linear maps $d(b) \in \operatorname{Hom}_{\mathbb{C}}\left(x_{s(b)}^{\prime}, x_{e(b)}^{\prime \prime}\right)$ such that

$$
\sum_{a \in Q_{1}: s(a)=p}\left(d(\bar{a}) x^{\prime}(a)+x^{\prime \prime}(\bar{a}) d(a)\right)-\sum_{a \in Q_{1}: e(a)=p}\left(d(a) x^{\prime}(\bar{a})+x^{\prime \prime}(a) d(\bar{a})\right)=0
$$

for all $p \in Q_{0}$. We call the elements in $D_{\Lambda}\left(x^{\prime}, x^{\prime \prime}\right)$ derivations.

Let $d=(d(a))_{a \in \bar{Q}_{1}}$ with $d(a) \in \operatorname{Hom}_{\mathbb{C}}\left(x_{s(a)}^{\prime}, x_{e(a)}^{\prime \prime}\right)$, and for each $a \in \bar{Q}_{1}$ let

$$
E_{d}(a)=\left(\begin{array}{cc}
x^{\prime}(a) & 0 \\
d(a) & x^{\prime \prime}(a)
\end{array}\right) .
$$

Then $E_{d}=\left(E_{d}(a)\right)_{a \in \bar{Q}_{1}}$ defines a $\Lambda$-module if and only if the maps $d(a)$ satisfy (5.1) for all $p$. In this case we obtain an obvious short exact sequence

$$
\epsilon_{d}: 0 \rightarrow x^{\prime \prime} \rightarrow E_{d} \rightarrow x^{\prime} \rightarrow 0
$$

\subsection{Inner derivations}

For $\Lambda$-modules $x^{\prime}$ and $x^{\prime \prime}$ let $I_{\Lambda}\left(x^{\prime}, x^{\prime \prime}\right)$ be the vector space of all tuples $i=(i(b))_{b \in \bar{Q}_{1}}$ of linear maps $i(b) \in \operatorname{Hom}_{\mathbb{C}}\left(x_{s(b)}^{\prime}, x_{e(b)}^{\prime \prime}\right)$ such that for some $\left(\phi_{q}\right)_{q \in Q_{0}}$ with $\phi_{q} \in \operatorname{Hom}_{\mathbb{C}}\left(x_{q}^{\prime}, x_{q}^{\prime \prime}\right)$ we have

$$
i(b)=\phi_{e(b)} x^{\prime}(b)-x^{\prime \prime}(b) \phi_{s(b)}
$$

for all $b \in \bar{Q}_{1}$. The elements in $I_{\Lambda}\left(x^{\prime}, x^{\prime \prime}\right)$ are called inner derivations and we obviously have $I_{\Lambda}\left(x^{\prime}, x^{\prime \prime}\right) \subseteq D_{\Lambda}\left(x^{\prime}, x^{\prime \prime}\right)$. Let

$$
\pi: D_{\Lambda}\left(x^{\prime}, x^{\prime \prime}\right) \rightarrow \operatorname{Ext}_{\Lambda}^{1}\left(x^{\prime}, x^{\prime \prime}\right)
$$

be defined by $\pi(d)=\left[\epsilon_{d}\right]$. It is known that the kernel of $\pi$ is just the set $I_{\Lambda}\left(x^{\prime}, x^{\prime \prime}\right)$ of inner derivations, hence we obtain an exact sequence

$$
0 \rightarrow \operatorname{Hom}_{\Lambda}\left(x^{\prime}, x^{\prime \prime}\right) \rightarrow \bigoplus_{q \in Q_{0}} \operatorname{Hom}_{\mathbb{C}}\left(x_{q}^{\prime}, x_{q}^{\prime \prime}\right) \rightarrow D_{\Lambda}\left(x^{\prime}, x^{\prime \prime}\right) \stackrel{\pi}{\rightarrow} \operatorname{Ext}_{\Lambda}^{1}\left(x^{\prime}, x^{\prime \prime}\right) \rightarrow 0 .
$$

We choose a fixed vector space decomposition

$$
D_{\Lambda}\left(x^{\prime}, x^{\prime \prime}\right)=I_{\Lambda}\left(x^{\prime}, x^{\prime \prime}\right) \oplus E_{\Lambda}\left(x^{\prime}, x^{\prime \prime}\right) .
$$




\section{Geiss, B. Leclerc And J. Schröer}

We can therefore identify $\operatorname{Ext}_{\Lambda}^{1}\left(x^{\prime}, x^{\prime \prime}\right)$ with $E_{\Lambda}\left(x^{\prime}, x^{\prime \prime}\right)$. We also can identify $\mathbb{P} \operatorname{Ext}_{\Lambda}^{1}\left(x^{\prime}, x^{\prime \prime}\right)$ with $\mathbb{P} E_{\Lambda}\left(x^{\prime}, x^{\prime \prime}\right)$. Set

$$
E_{\Lambda}^{*}\left(x^{\prime}, x^{\prime \prime}\right)=E_{\Lambda}\left(x^{\prime}, x^{\prime \prime}\right) \backslash\{0\}
$$

\subsection{Principal $\mathbb{C}^{*}$-bundles}

Recall that the multiplicative group $\mathbb{C}^{*}$ is special in the sense that each principal $\mathbb{C}^{*}$-bundle is locally trivial in the Zariski topology, see [Ser58, $\S 4$ ]. Thus, if $\pi: P \rightarrow Q$ is such a bundle, $\pi$ admits local sections. Since, moreover, the action of $\mathbb{C}^{*}$ on $P$ is free by definition, we conclude that $(\pi, Q)$ is a geometric quotient for the action of $\mathbb{C}^{*}$ on $P$; see, for example, [Bon98, Lemma 5.6]. Note, moreover, that $\pi$ is flat (and, in particular, open) since locally it is just a projection.

As a rule, we write $\mathbb{C}^{*} x$ for the $\mathbb{C}^{*}$-orbit of $x$ if $x$ belongs to a principal $\mathbb{C}^{*}$-bundle.

\subsection{The varieties $E F_{\mathrm{i}}\left(x^{\prime}, x^{\prime \prime}\right)$}

Let $x^{\prime} \in \Lambda_{\mathbf{V}^{\prime}}$ and $x^{\prime \prime} \in \Lambda_{\mathbf{V}^{\prime \prime}}$ and $\mathbf{i}=\left(i_{1}, i_{2}, \ldots, i_{m}\right)$ be a word in $I^{m}$ such that $|\mathbf{i}|=\underline{\operatorname{dim}}\left(x^{\prime} \oplus x^{\prime \prime}\right)$. The action of $\mathbb{C}^{*}$ on $\mathbf{V}^{\prime} \oplus \mathbf{V}^{\prime \prime}$ defined by

$$
\lambda \star\left(v^{\prime}, v^{\prime \prime}\right):=\left(v^{\prime}, \lambda \cdot v^{\prime \prime}\right)
$$

induces an action on the flag variety $\Phi_{\mathbf{i}}\left(\mathbf{V}^{\prime} \oplus \mathbf{V}^{\prime \prime}\right)$ : if

$$
x^{\bullet}=\left(x^{0} \supseteq x^{1} \supseteq \cdots \supseteq x^{m}\right),
$$

then the $i$ th component of $\left(\lambda \star x^{\bullet}\right)$ is $\left\{\lambda \star z \mid z \in x^{i}\right\}$.

On the other hand we have the principal $\mathbb{C}^{*}$-bundle $E_{\Lambda}^{*}\left(x^{\prime}, x^{\prime \prime}\right) \rightarrow \mathbb{P} E_{\Lambda}\left(x^{\prime}, x^{\prime \prime}\right)$. Thus, we obtain by $[$ Ser $58, \S 3.2]$ a new principal $\mathbb{C}^{*}$-bundle

$$
\tilde{q}: E_{\Lambda}^{*}\left(x^{\prime}, x^{\prime \prime}\right) \times \Phi_{\mathbf{i}}\left(\mathbf{V}^{\prime} \oplus \mathbf{V}^{\prime \prime}\right) \rightarrow E_{\Lambda}^{*}\left(x^{\prime}, x^{\prime \prime}\right) \times \mathbb{C}^{*} \Phi_{\mathbf{i}}\left(\mathbf{V}^{\prime} \oplus \mathbf{V}^{\prime \prime}\right) .
$$

We consider

$$
\widetilde{E F_{\mathbf{i}}}\left(x^{\prime}, x^{\prime \prime}\right):=\left\{\left(d, x^{\bullet}\right) \in E_{\Lambda}^{*}\left(x^{\prime}, x^{\prime \prime}\right) \times \Phi_{\mathbf{i}}\left(\mathbf{V}^{\prime} \oplus \mathbf{V}^{\prime \prime}\right) \mid x^{\bullet} \in \Phi_{\mathbf{i}, E_{d}}\right\} .
$$

This is clearly a closed subset of $E_{\Lambda}^{*}\left(x^{\prime}, x^{\prime \prime}\right) \times \Phi_{\mathbf{i}}\left(\mathbf{V}^{\prime} \oplus \mathbf{V}^{\prime \prime}\right)$, and it is moreover $\mathbb{C}^{*}$-stable since

$$
\left(\begin{array}{cc}
\mathbb{1}_{\mathbf{V}^{\prime}} & 0 \\
0 & \lambda \mathbb{1}_{\mathbf{V}^{\prime \prime}}
\end{array}\right): E_{d}=\left(\begin{array}{cc}
x^{\prime} & 0 \\
d & x^{\prime \prime}
\end{array}\right) \rightarrow E_{\lambda d}=\left(\begin{array}{cc}
x^{\prime} & 0 \\
\lambda d & x^{\prime \prime}
\end{array}\right)
$$

is an isomorphism of $\Lambda$-modules. We conclude that

$$
E F_{\mathbf{i}}\left(x^{\prime}, x^{\prime \prime}\right):=\tilde{q}\left(\widetilde{E F}_{\mathbf{i}}\left(x^{\prime}, x^{\prime \prime}\right)\right)
$$

is closed in $E_{\Lambda}^{*}\left(x^{\prime}, x^{\prime \prime}\right) \times \mathbb{C}^{*} \Phi_{\mathbf{i}}\left(\mathbf{V}^{\prime} \oplus \mathbf{V}^{\prime \prime}\right)$ since $\tilde{q}$ is open; see $\S 5$.3. Thus, the restriction of $\tilde{q}$ to

$$
q: \widetilde{E F_{\mathbf{i}}}\left(x^{\prime}, x^{\prime \prime}\right) \rightarrow E F_{\mathbf{i}}\left(x^{\prime}, x^{\prime \prime}\right)
$$

is again a principal $\mathbb{C}^{*}$-bundle $[\operatorname{Ser} 58, \S 3.1]$ and, in particular, $\left(q, E F_{\mathbf{i}}\left(x^{\prime}, x^{\prime \prime}\right)\right)$ is a geometric quotient for the action of $\mathbb{C}^{*}$ on $\widetilde{E F} \mathbf{i}\left(x^{\prime}, x^{\prime \prime}\right)$.

The projection

$$
\widetilde{p}_{1}: \widetilde{E F} \mathbf{i}\left(x^{\prime}, x^{\prime \prime}\right) \rightarrow \mathbb{P} E_{\Lambda}\left(x^{\prime}, x^{\prime \prime}\right),\left(d, x^{\bullet}\right) \mapsto \mathbb{C}^{*} d
$$

is constant on $\mathbb{C}^{*}$-orbits. So we obtain a morphism $p_{1}: E F_{\mathbf{i}}\left(x^{\prime}, x^{\prime \prime}\right) \rightarrow \mathbb{P} E_{\Lambda}\left(x^{\prime}, x^{\prime \prime}\right)$ which maps $\mathbb{C}^{*}\left(d, x^{\bullet}\right)$ to $\mathbb{C}^{*} d$. In other words, we have

$$
p_{1}^{-1}\left(\mathbb{C}^{*} d\right) \cong \Phi_{\mathbf{i}, E_{d}} .
$$




\subsection{The $\delta$-stratification of $E F_{\mathrm{i}}\left(\boldsymbol{x}^{\prime}, \boldsymbol{x}^{\prime \prime}\right)$}

Let $\mathbf{e}:=\underline{\operatorname{dim}}\left(x^{\prime}\right)+\underline{\operatorname{dim}}\left(x^{\prime \prime}\right)$ and consider the $\delta$-stratification

$$
\Lambda_{\mathbf{e}}=\bigsqcup_{x \in R(\mathbf{e})}\langle x\rangle .
$$

We claim that

$$
E F_{\mathbf{i}}\left(x^{\prime}, x^{\prime \prime}\right)_{\langle x\rangle}:=\left\{\mathbb{C}^{*}\left(d, x^{\bullet}\right) \in E F_{\mathbf{i}}\left(x^{\prime}, x^{\prime \prime}\right) \mid E_{d} \in\langle x\rangle\right\}
$$

is a constructible set. Indeed, we have a morphism $\iota: E_{\Lambda}\left(x^{\prime}, x^{\prime \prime}\right) \rightarrow \Lambda_{\mathbf{e}}$ which maps $d$ to $E_{d}$. Since $\langle x\rangle$ is constructible and $\mathrm{GL}_{\mathbf{e}}$-invariant,

$$
E_{\Lambda}\left(x^{\prime}, x^{\prime \prime}\right)_{\langle x\rangle}=\left\{d \in E_{\Lambda}\left(x^{\prime}, x^{\prime \prime}\right) \mid E_{d} \in\langle x\rangle\right\}
$$

is constructible and $\mathbb{C}^{*}$-invariant (see also (5.2)). Now,

$$
E F_{\mathbf{i}}\left(x^{\prime}, x^{\prime \prime}\right)_{\langle x\rangle}=p_{1}^{-1}\left(\mathbb{P} E_{\Lambda}\left(x^{\prime}, x^{\prime \prime}\right)_{\langle x\rangle}\right)
$$

is also constructible.

The fibers $p_{1}^{-1}([d])$ are identified with the varieties $\Phi_{\mathbf{i}, E_{d}}$. Since they all come from the same $\delta$-stratum, they all have the same Euler characteristic. Thus, from Proposition 7.4.1 we obtain

$$
\chi\left(E F_{\mathbf{i}}\left(x^{\prime}, x^{\prime \prime}\right)_{\langle x\rangle}\right)=\chi\left(\mathbb{P} E_{\Lambda}\left(x^{\prime}, x^{\prime \prime}\right)_{\langle x\rangle}\right) \cdot \chi\left(\Phi_{\mathbf{i}, E_{d}}\right)=\chi\left(\mathbb{P} \operatorname{Ext}_{\Lambda}^{1}\left(x^{\prime}, x^{\prime \prime}\right)_{\langle x\rangle}\right) \cdot \chi\left(\Phi_{\mathbf{i}, E_{d}}\right)
$$

for any $d \in E_{\Lambda}\left(x^{\prime}, x^{\prime \prime}\right)\langle x\rangle$. It follows that,

$$
\chi\left(E F_{\mathbf{i}}\left(x^{\prime}, x^{\prime \prime}\right)\right)=\sum_{x \in R(\mathbf{e})} \chi\left(\mathbb{P} \operatorname{Ext}_{\Lambda}^{1}\left(x^{\prime}, x^{\prime \prime}\right)_{\langle x\rangle}\right) \cdot \chi\left(\Phi_{\mathbf{i}, x}\right) .
$$

\subsection{Proof of Theorem 1}

We define a morphism of varieties

$$
\pi: E F_{\mathbf{i}}\left(x^{\prime}, x^{\prime \prime}\right) \rightarrow \bigsqcup_{\left(\mathbf{c}^{\prime}, \mathbf{c}^{\prime \prime}\right) \in W_{\mathbf{V}^{\prime}, \mathbf{V}^{\prime \prime}}}\left(\mathbb{P} E_{\Lambda}\left(x^{\prime}, x^{\prime \prime}\right) \times \Phi_{\mathbf{i}, \mathbf{c}^{\prime}, x^{\prime}} \times \Phi_{\mathbf{i}, \mathbf{c}^{\prime \prime}, x^{\prime \prime}}\right)
$$

which maps $\mathbb{C}^{*}\left(d, x^{\bullet}\right)$ to $\left(\mathbb{C}^{*} d, \mathfrak{f}_{x^{\prime}}, \mathfrak{f}_{x^{\prime \prime}}\right)$ where $\left(\mathfrak{f}_{x^{\prime}}, \mathfrak{f}_{x^{\prime \prime}}\right)$ is the pair of flags in $x^{\prime}$ and $x^{\prime \prime}$ induced by $x^{\bullet}$ via the short exact sequence

$$
\epsilon_{d}: 0 \rightarrow x^{\prime \prime} \rightarrow E_{d} \rightarrow x^{\prime} \rightarrow 0 .
$$

(Observe that $\left(d, x^{\bullet}\right)$ and $\left(\lambda d, \lambda \star x^{\bullet}\right)$ induce the same pair of flags via the sequences $\epsilon_{d}$ and $\epsilon_{\lambda d}$, respectively.) We denote by $L^{1}$ the image of $\pi$, and by $L^{2}$ the complement of $L^{1}$ in

$$
\bigsqcup_{\left(\mathbf{c}^{\prime}, \mathbf{c}^{\prime \prime}\right) \in W_{\mathbf{V}^{\prime}, \mathbf{V}^{\prime \prime}}}\left(\mathbb{P} E_{\Lambda}\left(x^{\prime}, x^{\prime \prime}\right) \times \Phi_{\mathbf{i}, \mathbf{c}^{\prime}, x^{\prime}} \times \Phi_{\mathbf{i}, \mathbf{c}^{\prime \prime}, x^{\prime \prime}}\right) .
$$

The following diagram illustrates the situation.

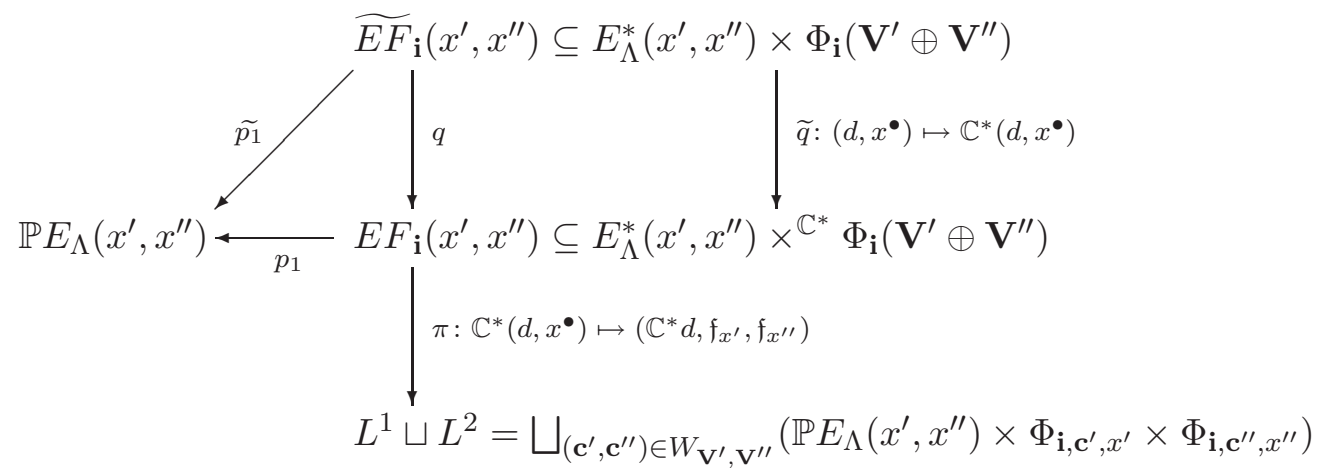




\section{Geiss, B. Leclerc And J. Schröer}

Proposition 5.6.1. The following hold:

(a) $\chi\left(L^{1}\right)=\chi\left(E F_{\mathbf{i}}\left(x^{\prime}, x^{\prime \prime}\right)\right)$;

(b) $\chi\left(\bigsqcup_{\left(\mathbf{c}^{\prime}, \mathbf{c}^{\prime \prime}\right) \in W_{\mathbf{V}^{\prime}, \mathbf{v}^{\prime \prime}}}\left(\mathbb{P} E_{\Lambda}\left(x^{\prime}, x^{\prime \prime}\right) \times \Phi_{\mathbf{i}, \mathbf{c}^{\prime}, x^{\prime}} \times \Phi_{\mathbf{i}, \mathbf{c}^{\prime \prime}, x^{\prime \prime}}\right)\right)=\chi\left(\mathbb{P} E_{\Lambda}\left(x^{\prime}, x^{\prime \prime}\right)\right) \cdot \chi\left(\Phi_{\mathbf{i}, x^{\prime} \oplus x^{\prime \prime}}\right)$;

(c) $\chi\left(L^{2}\right)=\chi\left(E F_{\mathbf{i}}\left(x^{\prime \prime}, x^{\prime}\right)\right)$.

Proof. (a) By the same argument as in Lemma 3.3.1, the fibers of $\pi$ are isomorphic to affine spaces. This implies part (a) by Proposition 7.4.1.

(b) We have to show that

$$
\sum_{\left(\mathbf{c}^{\prime}, \mathbf{c}^{\prime \prime}\right) \in W_{\mathbf{V}^{\prime}, \mathbf{V}^{\prime \prime}}} \chi\left(\Phi_{\mathbf{i}, \mathbf{c}^{\prime}, x^{\prime}}\right) \cdot \chi\left(\Phi_{\mathbf{i}^{\prime \prime}, \mathbf{c}^{\prime \prime}, x^{\prime \prime}}\right)=\chi\left(\Phi_{\mathbf{i}, x^{\prime} \oplus x^{\prime \prime}}\right) .
$$

This is explained in the proof of [GLS05a, Lemma 6.1], see also the remark after Corollary 3.3.3.

(c) Let $\left(\mathbb{P}[d], \mathfrak{f}_{x^{\prime}}, \mathfrak{f}_{x^{\prime \prime}}\right) \in L^{2}$. By Lemma 2.4.2, this means that for all $\left(\mathbf{c}^{\prime}, \mathbf{c}^{\prime \prime}\right) \in W_{\mathbf{V}^{\prime}, \mathbf{V}^{\prime \prime}}$,

$$
d \notin p_{0}\left(\operatorname{Ker}\left(\beta_{\mathbf{i}, \mathbf{c}^{\prime}, \mathbf{c}^{\prime \prime}, \mathfrak{f}_{x^{\prime}}, \mathfrak{f}_{x^{\prime \prime}}}^{\prime}\right)\right) \text {. }
$$

By Proposition 2.5.2, this means that for all $\left(\mathbf{c}^{\prime}, \mathbf{c}^{\prime \prime}\right) \in W_{\mathbf{V}^{\prime}, \mathbf{V}^{\prime \prime}}$,

$$
d \not \perp\left(E_{\Lambda}\left(x^{\prime \prime}, x^{\prime}\right) \cap \operatorname{Im}\left(\beta_{\mathbf{i}, \mathbf{c}^{\prime \prime}, \mathbf{c}^{\prime}, \mathfrak{f}_{x^{\prime \prime}}, \mathfrak{f}_{x^{\prime}}}\right)\right) .
$$

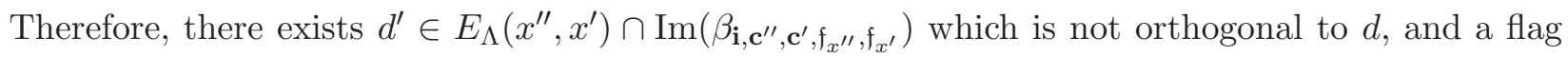
$y^{\bullet}$ of submodules of the module

$$
\left(\begin{array}{cc}
x^{\prime \prime} & 0 \\
d^{\prime} & x^{\prime}
\end{array}\right)
$$

such that $y^{\bullet}$ induces $\mathfrak{f}_{x^{\prime \prime}}$ and $\mathfrak{f}_{x^{\prime}}$. We are thus led to consider the constructible set $C$ of all pairs

$$
\left(\left(\mathbb{C}^{*} d, \mathfrak{f}_{x^{\prime}}, \mathfrak{f}_{x^{\prime \prime}}\right), \mathbb{C}^{*}\left(d^{\prime}, y^{\bullet}\right)\right) \in L^{2} \times E F_{\mathbf{i}}\left(x^{\prime \prime}, x^{\prime}\right)
$$

such that $\left(d^{\prime}, y^{\bullet}\right)$ induces $\left(\mathfrak{f}_{x^{\prime \prime}}, \mathfrak{f}_{x^{\prime}}\right)$, and $d$ and $d^{\prime}$ are not orthogonal for the pairing between $E_{\Lambda}\left(x^{\prime}, x^{\prime \prime}\right)$ and $E_{\Lambda}\left(x^{\prime \prime}, x^{\prime}\right)$. Let us consider the two natural projections as follows.

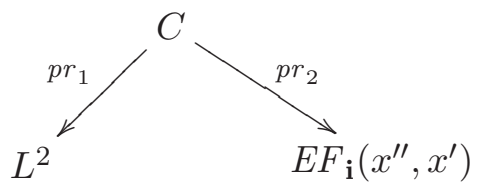

We are going to show that all fibers of both projections have Euler characteristic equal to 1. More precisely, we have:

(i) the map $p r_{1}$ is surjective with fibers being extensions of affine spaces;

(ii) the map $p r_{2}$ is surjective with fibers being affine spaces (of constant dimension $\operatorname{dim} E_{\Lambda}\left(x^{\prime \prime}, x^{\prime}\right)$ $-1)$.

Let us prove part (i). Let $\left(\mathbb{C}^{*} d, \mathfrak{f}_{x^{\prime}}, \mathfrak{f}_{x^{\prime \prime}}\right) \in L^{2}$. Let $E_{\Lambda}\left(x^{\prime \prime}, x^{\prime}\right)_{\left(\mathfrak{f}_{x^{\prime \prime}}, \mathfrak{f}_{x^{\prime}}\right)}$ be the set of all $d^{\prime} \in E_{\Lambda}\left(x^{\prime \prime}, x^{\prime}\right)$ such that there exists a filtration $y^{\bullet}$ of the module

$$
\left(\begin{array}{cc}
x^{\prime \prime} & 0 \\
d^{\prime} & x^{\prime}
\end{array}\right)
$$

which induces $\mathfrak{f}_{x^{\prime \prime}}$ and $\mathfrak{f}_{x^{\prime}}$. This is a vector space, and by the above discussion we know that it is not contained in the hyperplane $d^{\perp}$. Thus,

$$
d^{\perp} \cap E_{\Lambda}\left(x^{\prime \prime}, x^{\prime}\right)_{\left(\mathfrak{f}_{x^{\prime \prime}}, \mathfrak{f}_{x^{\prime}}\right)}
$$

is a hyperplane in $E_{\Lambda}\left(x^{\prime \prime}, x^{\prime}\right)_{\left(\mathfrak{f}_{x^{\prime \prime}}, \mathfrak{f}_{x^{\prime}}\right)}$, and

$$
Z:=\mathbb{P}\left(E_{\Lambda}\left(x^{\prime \prime}, x^{\prime}\right)_{\left(\mathfrak{f}_{x^{\prime \prime}}, \mathfrak{f}_{x^{\prime}}\right)} \backslash d^{\perp}\right)
$$


is an affine space. We get a map

$$
\operatorname{pr}_{1}^{-1}\left(\mathbb{C}^{*} d, \mathfrak{f}_{x^{\prime}}, \mathfrak{f}_{x^{\prime \prime}}\right) \rightarrow Z
$$

which maps $\left(\left(\mathbb{C}^{*} d, \mathfrak{f}_{x^{\prime}}, \mathfrak{f}_{x^{\prime \prime}}\right), \mathbb{C}^{*}\left(d^{\prime}, y^{\bullet}\right)\right)$ to $\mathbb{C}^{*} d^{\prime}$. By construction this map is surjective and its fibers are affine spaces which implies part (i).

Let us prove part (ii). Let $\mathbb{C}^{*}\left(d^{\prime}, y^{\bullet}\right) \in E F_{\mathbf{i}}\left(x^{\prime \prime}, x^{\prime}\right)$. Let $\mathfrak{f}_{x^{\prime \prime}}$ and $\mathfrak{f}_{x^{\prime}}$ be the flags induced by $y^{\bullet}$ on $x^{\prime \prime}$ and $x^{\prime}$, respectively. Then, by Lemma 2.4.3 and Proposition 2.5.2,

$$
d^{\prime} \in p_{0}\left(\operatorname{Ker}\left(\beta_{\mathbf{i}, \mathbf{c}^{\prime}, \mathbf{c}^{\prime \prime}, \mathfrak{f}_{x^{\prime}}, \mathfrak{f}_{x^{\prime \prime}}}^{\prime}\right)\right)^{\perp}
$$

for some $\left(\mathbf{c}^{\prime}, \mathbf{c}^{\prime \prime}\right) \in W_{\mathbf{V}^{\prime}, \mathbf{V}^{\prime \prime}}$. So if $d \notin \perp d^{\prime}$, then $\left(\mathbb{C}^{*} d, \mathfrak{f}_{x^{\prime}}, \mathfrak{f}_{x^{\prime \prime}}\right) \in L^{2}$, by Lemma 2.4.2. Therefore, $\operatorname{pr}_{2}^{-1}\left(\left[d^{\prime}, y^{\bullet}\right]\right)$ can be identified with the projectivization of the set of all $d \in E_{\Lambda}\left(x^{\prime}, x^{\prime \prime}\right)$ such that $d \notin{ }^{\perp} d^{\prime}$. However, the projectivization of the complement of a hyperplane in a vector space of dimension $m$ is an affine space of dimension $m-1$.

Now, the proposition together with (5.3) obviously implies (2.1), which is equivalent to Theorem 1.

\section{An example}

In this section, $\Lambda$ will be the preprojective algebra of type $D_{4}$, with the following underlying quiver.

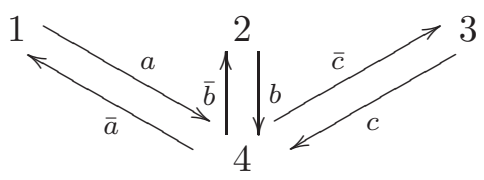

We study extensions between

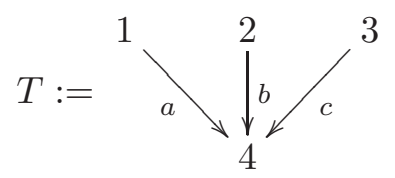

and the simple module $S_{4}$. We have $\operatorname{dim} \operatorname{Ext}_{\Lambda}^{1}\left(T, S_{4}\right)=2$. The middle terms of the non-split short exact sequences

$$
0 \rightarrow T \rightarrow E \rightarrow S_{4} \rightarrow 0
$$

are of the following form.

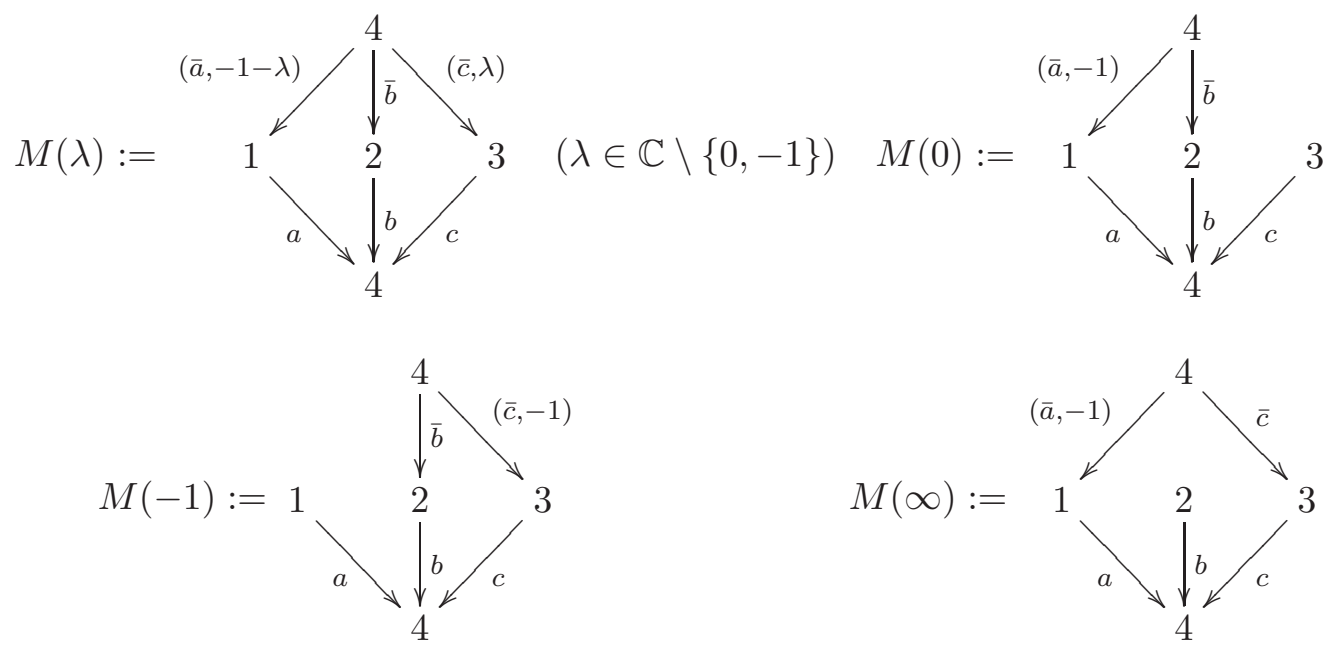




\section{Geiss, B. LeClerc And J. Schröer}

The middle terms of the non-split short exact sequences

$$
0 \rightarrow S_{4} \rightarrow E \rightarrow T \rightarrow 0
$$

are of the following form.

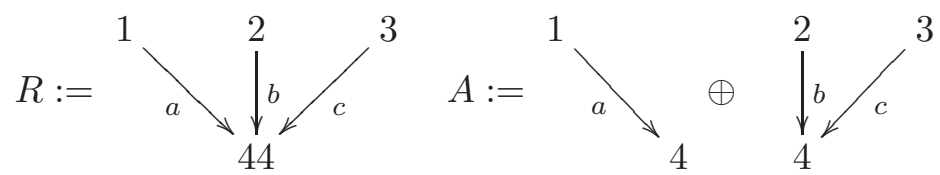

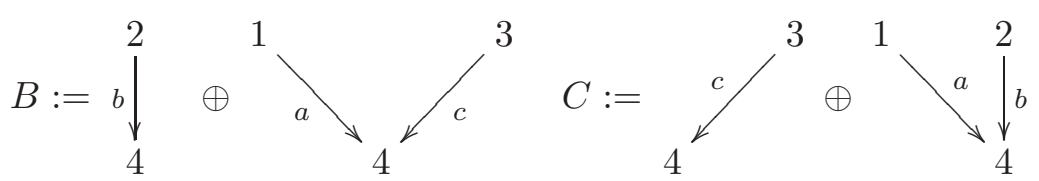

Note that $R$ is rigid, and $A, B, C$ belong to the orbit closure of $R$. Our multiplication formula yields, for any $\lambda \in \mathbb{C} \backslash\{0,-1\}$,

$$
2 \delta_{T} \cdot \delta_{S_{4}}=\left((-1) \delta_{M(\lambda)}+\delta_{M(0)}+\delta_{M(-1)}+\delta_{M(\infty)}\right)+\left((-1) \delta_{R}+\delta_{A}+\delta_{B}+\delta_{C}\right) .
$$

To see this, note that $\operatorname{PExt}_{\Lambda}^{1}\left(S_{4}, T\right)$ is a projective line, with points identified to the middle terms of short exact sequences

$$
0 \rightarrow T \rightarrow M(\lambda) \rightarrow S_{4} \rightarrow 0 \quad \text { with } \lambda \in \mathbb{C} \cup\{\infty\}
$$

This becomes 'stratified' according to the Euler characteristics of flags of submodules into $(\mathbb{C} \backslash$

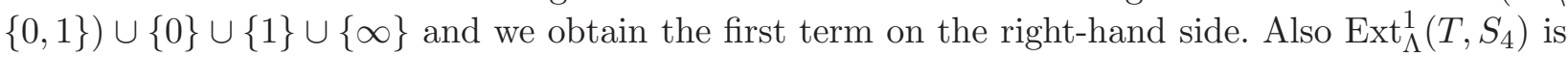
a projective line with three special points corresponding to the extensions with middle term $A, B$, $C$ and generic points corresponding to the extensions with middle term $R$. This gives the second term on the right-hand side of our equation.

Using the rigid modules
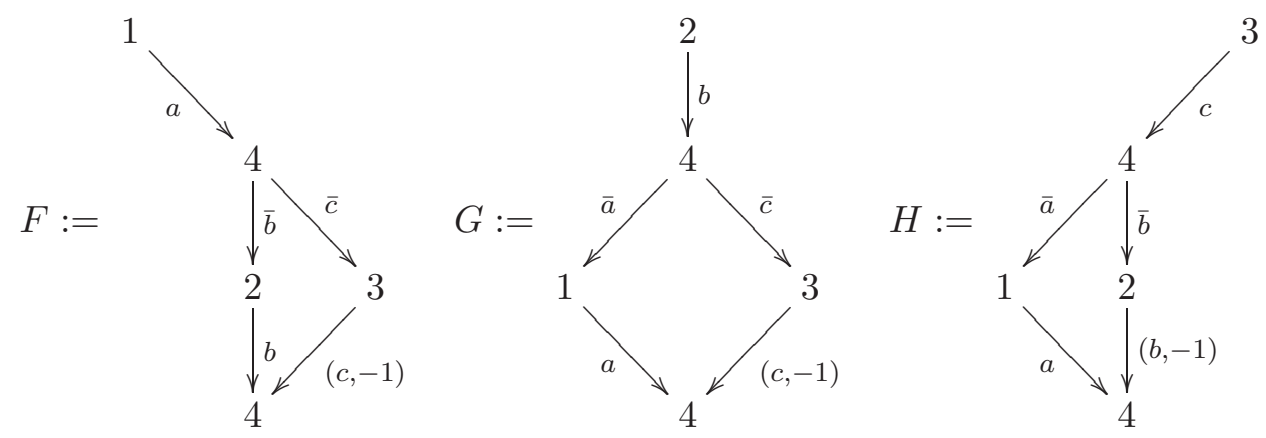

we obtain

$$
\begin{aligned}
\delta_{M(0)}=\delta_{M(\lambda)}+\delta_{H}, & \delta_{A}=\delta_{R}+\delta_{F}, \\
\delta_{M(-1)}=\delta_{M(\lambda)}+\delta_{F}, & \delta_{B}=\delta_{R}+\delta_{G}, \\
\delta_{M(\infty)}=\delta_{M(\lambda)}+\delta_{G}, & \delta_{C}=\delta_{R}+\delta_{H} .
\end{aligned}
$$

These equalities follow from simple calculations of Euler characteristics of varieties of composition series. For example, to see the first equality one should observe that for $M(0)$ there are two types of composition series. Those with top $S_{4}$ may be identified with the composition series of $M(\lambda)$ for $\lambda$ 'generic'. The remaining composition series have top $S_{3}$ and may be identified with the composition series of $H$. Our claim follows, see $\S 2.1$. 
So, eventually,

$$
\delta_{T} \cdot \delta_{S_{4}}=\delta_{M(\lambda)}+\delta_{R}+\delta_{F}+\delta_{G}+\delta_{H}
$$

as an expansion in the dual semicanonical basis.

\section{Recollections}

\subsection{The 2-Calabi-Yau algebras and categories}

Let $\mathrm{k}$ be a field. A triangulated k-category $\mathcal{T}$ with suspension functor $\Sigma$ is called a $d$-Calabi-Yau category if all its homomorphism spaces are finite-dimensional over $\mathrm{k}$, and there exists a functorial isomorphism

$$
\mathcal{T}(x, y) \rightarrow \operatorname{Hom}_{\mathrm{k}}\left(\mathcal{T}\left(y, \Sigma^{d} x\right), \mathrm{k}\right)
$$

for all $x, y \in \mathcal{T}$.

It is well known that for a preprojective algebra $\Lambda$ of Dynkin type the stable module category $\Lambda$ - $\underline{\bmod }$ with the suspension functor $\Omega^{-1}$ is a 2-Calabi-Yau category; see, for example, [GLS05b, $\S 7.5]$. In this case, (1.1) follows via the natural isomorphism (see [Hel60, §6]) $\operatorname{Ext}_{\Lambda}^{1}(x, y) \rightarrow$ $\underline{\operatorname{Hom}}_{\Lambda}\left(x, \Omega^{-1} y\right)$ from the 2 -Calabi-Yau property of $\Lambda$ - $\underline{\bmod }$.

Recently it was announced by Chuang and Rouquier (see also remark (1) in $\S 8.3$ ) that a (connected) preprojective algebra $\Lambda$ which is not of Dynkin type is a 2 -Calabi-Yau algebra, so by definition $\mathcal{D}_{f}(\Lambda)$, the full subcategory of the bounded derived category of $\Lambda$ of complexes with finite-dimensional nilpotent cohomology, is a 2-Calabi-Yau category. In this case, (1.1) follows from the 2-Calabi-Yau property of $\mathcal{D}_{f}(\Lambda)$ via the natural isomorphism $\operatorname{Ext}_{\Lambda}^{1}(x, y) \rightarrow \mathcal{D}_{f}(x, y[1])$ for nilpotent $\Lambda$-modules $x, y$.

\subsection{Extensions}

If $\epsilon: 0 \rightarrow y^{\prime \prime} \rightarrow y \rightarrow y^{\prime} \rightarrow 0$ is a short exact sequence of $\Lambda$-modules, we write $[\epsilon]$ for its class in $\operatorname{Ext}_{\Lambda}^{1}\left(y^{\prime}, y^{\prime \prime}\right)$. Let us recall the functorial behavior of $\operatorname{Ext}_{\Lambda}^{1}$ in terms of short exact sequences. If $\rho \in \operatorname{Hom}_{\Lambda}\left(x^{\prime}, y^{\prime}\right)$ and $\lambda \in \operatorname{Hom}_{\Lambda}\left(y^{\prime \prime}, z^{\prime \prime}\right)$, then $[\epsilon] \circ \rho \in \operatorname{Ext}_{\Lambda}^{1}\left(x^{\prime}, y^{\prime \prime}\right)$ is represented precisely by a short exact sequence $0 \rightarrow y^{\prime \prime} \rightarrow y \rightarrow x^{\prime} \rightarrow 0$ which is obtained from $\epsilon$ as the pullback along $\rho$. Similarly, $\lambda \circ[\epsilon] \in \operatorname{Ext}_{\Lambda}^{1}\left(y^{\prime}, z^{\prime \prime}\right)$ is represented precisely by a short exact sequence $0 \rightarrow z^{\prime \prime} \rightarrow z \rightarrow y^{\prime} \rightarrow 0$ which is obtained from $\epsilon$ as the pushout of $\epsilon$ along $\lambda$.

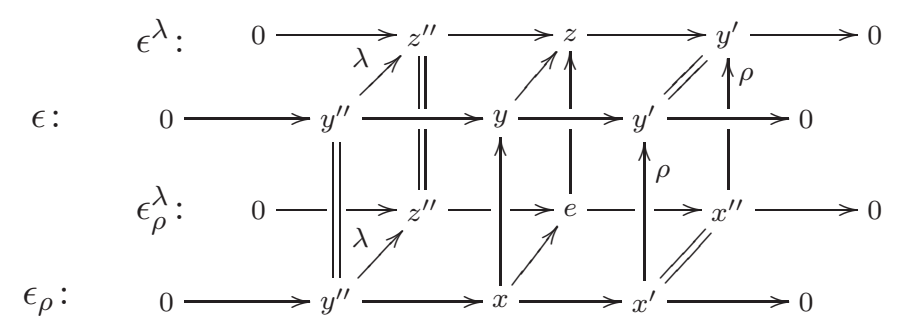

So in the above diagram we have $\left[\epsilon^{\lambda}\right]=\lambda \circ[\epsilon]$ and $\left[\epsilon_{\rho}\right]=[\epsilon] \circ \rho$ and the associativity $(\lambda \circ[\epsilon]) \circ \rho=$ $\left[\epsilon_{\rho}^{\lambda}\right]=\lambda \circ([\epsilon] \circ \rho)$.

\subsection{Bilinear forms and orthogonality}

Let $\phi: U \times U^{\prime} \rightarrow \mathbb{C}$ be a bilinear form. For subspaces $L \subseteq U$ and $L^{\prime} \subseteq U^{\prime}$ define

$$
\begin{aligned}
& L^{\perp}=\left\{u^{\prime} \in U^{\prime} \mid \phi\left(u, u^{\prime}\right)=0 \text { for all } u \in L\right\}, \\
& { }^{\perp} L^{\prime}=\left\{u \in U \mid \phi\left(u, u^{\prime}\right)=0 \text { for all } u^{\prime} \in L^{\prime}\right\} .
\end{aligned}
$$




\section{Geiss, B. Leclerc And J. Schröer}

We call $u \in U$ and $u^{\prime} \in U^{\prime}$ orthogonal (with respect to $\phi$ ) if $\phi\left(u, u^{\prime}\right)=0$. If $\phi$ is non-degenerate, then ${ }^{\perp}\left(L^{\perp}\right)=L$ and $\left({ }^{\perp} L^{\prime}\right)^{\perp}=L^{\prime}$, and

$$
\operatorname{dim} L+\operatorname{dim} L^{\perp}=\operatorname{dim} L^{\prime}+\operatorname{dim}^{\perp} L^{\prime}=\operatorname{dim} U=\operatorname{dim} U^{\prime} .
$$

Let $\phi_{V}: V \times V^{\prime} \rightarrow \mathbb{C}$ and $\phi_{W}: W \times W^{\prime} \rightarrow \mathbb{C}$ be non-degenerate bilinear forms, and let $f \in$ $\operatorname{Hom}_{\mathbb{C}}(V, W)$. Then we can identify $f$ with the dual $f^{\prime *}: V^{\prime *} \rightarrow W^{* *}$ of a map $f^{\prime} \in \operatorname{Hom}_{\mathbb{C}}\left(W^{\prime}, V^{\prime}\right)$ if and only if

$$
\phi_{V}\left(v, f^{\prime}\left(w^{\prime}\right)\right)=\phi_{W}\left(f(v), w^{\prime}\right)
$$

for all $v \in V$ and $w^{\prime} \in W^{\prime}$. Here we use the isomorphisms $\widetilde{\phi}_{V}: V \rightarrow V^{* *}$ and $\widetilde{\phi}_{W}: W \rightarrow W^{*}$ defined by $v \mapsto \phi_{V}(v,-)$ and $w \mapsto \phi_{W}(w,-)$, respectively. Assume that (7.1) holds. Thus, we get the following commutative diagram.
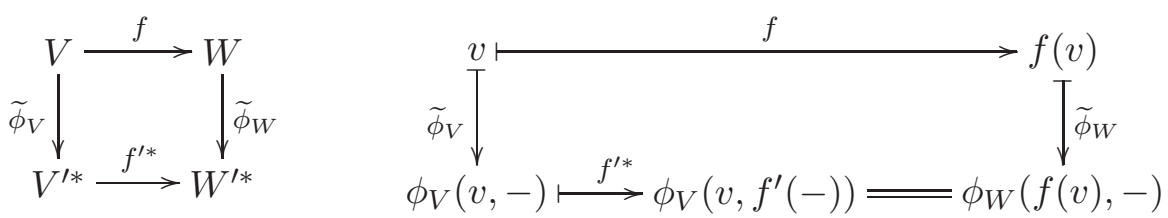

The proof of the following lemma is an easy exercise.

Lemma 7.3.1. We have $\operatorname{Ker}\left(f^{\prime}\right)=\operatorname{Im}(f)^{\perp}$.

\subsection{Euler characteristics}

For a complex algebraic variety $V$, let $\mathcal{C}(V)$ denote the abelian group of constructible functions on $V$ with respect to the Zariski topology.

If $\pi: V \rightarrow W$ is a morphism of complex varieties and $f \in \mathcal{C}(V)$, we define a function $\pi_{*} f$ on $W$ by

$$
\left(\pi_{*} f\right)(w)=\int_{\pi^{-1}(w)} f=\sum_{a \in \mathbb{C}} a \chi\left(f^{-1}(a) \cap \pi^{-1}(w)\right), \quad(w \in W) .
$$

Then it is known that $\pi_{*} f$ is constructible. Moreover, for morphisms $\pi: V \rightarrow W$ and $\theta: W \rightarrow U$ of complex varieties we have

$$
(\theta \circ \pi)_{*}=\theta_{*} \circ \pi_{*} .
$$

(The case of compact complex algebraic varieties is discussed in [Mac74, Proposition 1], the general case can be found in [Dim04, Proposition 4.1.31].) As a particular case, we note the following result.

Proposition 7.4.1. Let $\pi: V \rightarrow W$ be a morphism of complex varieties such that there exists some $c \in \mathbb{Z}$ with $\chi\left(\pi^{-1}(w)\right)=c$ for all $w \in \operatorname{Im}(\pi)$. Then

$$
\chi(V)=c \chi(\operatorname{Im}(\pi)) .
$$

In particular, if $\pi: V \rightarrow W$ is a morphism of complex varieties such that for all $w \in \operatorname{Im} \pi$ the fiber $\pi^{-1}(w)$ is isomorphic to an affine space, then $\chi(V)=\chi(\operatorname{Im}(\pi))$.

\section{Ext-symmetry for preprojective algebras}

The main goal of this section is to provide a direct proof of the following result which is crucial for this paper. Otherwise it is independent of the main body of the paper.

Theorem 3. For a quiver $Q$ without loops let $\Lambda$ be the associated preprojective algebra over a field $\mathrm{k}$. Then for all finite-dimensional $\Lambda$-modules $M$ and $N$ there is a functorial isomorphism

$$
\phi_{M, N}: \operatorname{Ext}_{\Lambda}^{1}(M, N) \rightarrow D \operatorname{Ext}_{\Lambda}^{1}(N, M) .
$$




\subsection{Preliminaries}

Let k be a field and let $Q=\left(Q_{0}, Q_{1}, s, e\right)$ be a finite quiver without loops. Here $Q_{0}$ denotes the set of vertices, $Q_{1}$ is the set of arrows of $Q$ and $s, e: Q_{1} \rightarrow Q_{0}$ are maps. An arrow $\alpha \in Q_{1}$ starts in a vertex $s \alpha=s(\alpha)$ and ends in a vertex $e \alpha=e(\alpha)$.

By $\bar{Q}$ we denote the double quiver of $Q$, so $\bar{Q}_{0}=Q_{0}, \bar{Q}_{1}=Q_{1} \cup\left\{\bar{\alpha} \mid \alpha \in Q_{1}\right\}$, and we extend the maps $s, e$ to maps $s, e: \bar{Q}_{1} \rightarrow Q_{0}$ by $s \bar{\alpha}:=e \alpha, e \bar{\alpha}:=s \alpha$ for all $\alpha \in Q_{1}$. It will be convenient to consider $\bar{?}$ as an involution on $\bar{Q}_{1}$ with

$$
\bar{\beta}:= \begin{cases}\bar{\beta} & \text { if } \beta \in Q_{1}, \\ \alpha & \text { if } \beta=\bar{\alpha} \text { for some } \alpha \in Q_{1} .\end{cases}
$$

Moreover, for all $\beta \in \bar{Q}_{1}$ we define

$$
|\beta|:= \begin{cases}0 & \text { if } \beta \in Q_{1} \\ 1 & \text { otherwise }\end{cases}
$$

We consider the preprojective algebra

$$
\Lambda=\mathrm{k} \bar{Q} /\left\langle\left.\rho_{i}\right|_{i \in Q_{0}}\right\rangle \quad \text { where } \rho_{i}=\sum_{\substack{\beta \in \bar{Q}_{1} \\ s \beta=i}}(-1)^{|\beta|} \bar{\beta} \beta .
$$

Note that $\Lambda$ is a quadratic, possibly infinite-dimensional quiver algebra; in any case $\Lambda$ is augmented over $S:=\mathrm{k}^{\times Q_{0}}$ (i.e. we have k-algebra homomorphisms $S \rightarrow \Lambda \rightarrow S$ whose composition is the identity on $S$ ). Note that $S$ is a commutative semisimple k-algebra which has a natural basis consisting of primitive orthogonal idempotents $\left\{e_{i} \mid i \in Q_{0}\right\}$. In what follows, all undecorated tensor products are meant over $S$.

The proof relies on the following well-known result which holds mutatis mutandis more generally for any quadratic quiver algebra. It follows, for example, from the proof of [BBK02, Theorem 3.15]. Lemma 8.1.1. Let $\Lambda$ be the preprojective algebra as defined above. Then

$$
P^{\bullet}: \Lambda \otimes R \otimes \Lambda \stackrel{d^{1}}{\longrightarrow} \Lambda \otimes A \otimes \Lambda \stackrel{d^{0}}{\longrightarrow} \Lambda \otimes S \otimes \Lambda
$$

is the beginning of a projective bimodule resolution of $\Lambda$, where $S=\bigoplus_{i \in Q_{0}} \mathrm{k} e_{i}, A=\bigoplus_{\beta \in \bar{Q}_{1}} \mathrm{k} \beta$ and $R=\bigoplus_{i \in Q_{0}} \mathrm{k} \rho_{i}$ are $S$-S-bimodules in the obvious way, and

$$
\begin{aligned}
& d^{0}=\sum_{\beta \in \bar{Q}_{1}}\left(\beta \otimes e_{s \beta} \otimes e_{s \beta}-e_{e \beta} \otimes e_{e \beta} \otimes \beta\right) \otimes_{S-S} \beta^{*}, \\
& d^{1}=\sum_{\beta \in \bar{Q}_{1}}(-1)^{|\beta|}\left(\bar{\beta} \otimes \beta \otimes e_{s \beta}+e_{s \beta} \otimes \bar{\beta} \otimes \beta\right) \otimes_{S-S} \rho_{s \beta}^{*} .
\end{aligned}
$$

In the statement of the lemma we denote, for example, the dual basis for the $S$ - $S$-bimodule $D A=\operatorname{Hom}_{\mathrm{k}}(A, \mathrm{k})$ by $\left(\beta^{*}\right)_{\beta \in \bar{Q}_{1}}$. In this case we have $e_{i} \beta^{*} e_{j}=\delta_{i, s \beta} \delta_{j, e \beta} \beta^{*}$.

\subsection{Proof of Theorem 3}

For finite-dimensional $\Lambda$-modules $M$ and $N$ it is easy to determine the complex $\operatorname{Hom}_{\Lambda}\left(P^{\bullet} \otimes_{\Lambda} M, N\right)$ :

$$
\bigoplus_{i \in Q_{0}} \operatorname{Hom}_{\mathrm{k}}(M(i), N(i)) \stackrel{d_{M, N}^{0}}{\longrightarrow} \bigoplus_{\beta \in \bar{Q}_{1}} \operatorname{Hom}_{\mathrm{k}}(M(s \beta), N(e \beta)) \stackrel{d_{M, N}^{1}}{\longrightarrow} \bigoplus_{i \in Q_{0}} \operatorname{Hom}_{\mathrm{k}}(M(i), N(i))
$$

where $d_{M, N}^{0}=\operatorname{Hom}_{\Lambda}\left(d^{0} \otimes_{\Lambda} M, N\right)$ is given by

$$
\left(f_{i}\right)_{i \in Q_{0}} \mapsto\left(N(\beta) f_{s \beta}-f_{e \beta} M(\beta)\right)_{\beta \in \bar{Q}_{1}}
$$




\section{Geiss, B. Leclerc And J. Schröer}

and $d_{M, N}^{1}=\operatorname{Hom}_{\Lambda}\left(d^{1} \otimes_{\Lambda} M, N\right)$ is given by

$$
\left(g_{\beta}\right)_{\beta \in \bar{Q}_{1}} \mapsto\left(\sum_{\beta \in \bar{Q}_{1}: s \beta=i}(-1)^{|\beta|}\left(N(\bar{\beta}) g_{\beta}+g_{\bar{\beta}} M(\beta)\right)\right)_{i \in Q_{0}} .
$$

Thus, we have functorially $\operatorname{Ext}_{\Lambda}^{1}(M, N)=\operatorname{Ker}\left(d_{M, N}^{1}\right) / \operatorname{Im}\left(d_{M, N}^{0}\right)$.

Similarly, we find $D \operatorname{Hom}_{\Lambda}\left(P^{\bullet} \otimes_{\Lambda} N, M\right)$, which is identified via the trace pairings

$$
\left(\bigoplus_{i \in Q_{0}} \operatorname{Hom}_{\mathrm{k}}(M(i), N(i))\right) \times\left(\bigoplus_{i \in Q_{0}} \operatorname{Hom}_{\mathrm{k}}(N(i), M(i))\right) \rightarrow \mathrm{k}, \quad\left(\left(\varphi_{i}\right),\left(f_{i}\right)\right) \mapsto \sum_{i \in Q_{0}} \operatorname{Tr}\left(\varphi_{i} \circ f_{i}\right)
$$

and

$$
\left(\bigoplus_{\beta \in \bar{Q}_{1}} \operatorname{Hom}_{\mathrm{k}}(M(s \beta), N(e \beta))\right) \times\left(\bigoplus_{\beta \in \bar{Q}_{1}} \operatorname{Hom}_{\mathrm{k}}(N(s \beta), M(e \beta))\right) \rightarrow \mathrm{k}, \quad\left(\left(\epsilon_{\beta}\right),\left(g_{\beta}\right)\right) \mapsto \sum_{\beta \in \bar{Q}_{1}} \operatorname{Tr}\left(\epsilon_{\bar{\beta}}^{\circ} \circ g_{\beta}\right)
$$

to

$$
\bigoplus_{i \in Q_{0}} \operatorname{Hom}_{\mathrm{k}}(M(i), N(i)) \stackrel{d_{N, M}^{1, *}}{\longrightarrow} \bigoplus_{\beta \in \bar{Q}_{1}} \operatorname{Hom}_{\mathrm{k}}(M(s \beta), N(e \beta)) \stackrel{d_{N, M}^{0, *}}{\longrightarrow} \bigoplus_{i \in Q_{0}} \operatorname{Hom}_{\mathrm{k}}(M(i), N(i))
$$

with $d_{N, M}^{1, *}=D \operatorname{Hom}_{\Lambda}\left(d^{1} \otimes N, M\right)$ given by

$$
\left(\varphi_{i}\right)_{i \in Q_{0}} \mapsto\left((-1)^{|\beta|}\left(N(\beta) \varphi_{s \beta}-\varphi_{e \beta} M(\beta)\right)\right)_{\beta \in \bar{Q}_{1}}
$$

and $d_{N, M}^{0, *}=D \operatorname{Hom}_{\Lambda}\left(d^{0} \otimes N, M\right)$ given by

$$
\left(\epsilon_{\beta}\right)_{\beta \in \bar{Q}_{1}} \mapsto\left(\sum_{\beta \in \bar{Q}_{1}: e \beta=i} \epsilon_{\beta} M(\bar{\beta})-\sum_{\beta \in \bar{Q}_{1}: s \beta=i} N(\bar{\beta}) \epsilon_{\beta}\right)_{i \in Q_{0}} .
$$

In fact, we have by definition

$$
\begin{aligned}
d_{N, M}^{1, *}\left(\left(\varphi_{i}\right)_{i \in Q_{0}}\right)\left(\left(g_{\beta}\right)_{\beta \in \bar{Q}_{1}}\right) & =\sum_{i \in Q_{0}} \operatorname{Tr}\left(\varphi_{i} \circ\left(\sum_{\substack{\beta \in \bar{Q}_{1} \\
s \beta=i}}(-1)^{|\beta|}\left(M(\bar{\beta}) g_{\beta}+g_{\bar{\beta}} N(\beta)\right)\right)\right) \\
& =\sum_{\beta \in \bar{Q}_{1}}(-1)^{|\beta|}\left(\operatorname{Tr}\left(\varphi_{e \bar{\beta}} M(\bar{\beta}) g_{\beta}\right)+\operatorname{Tr}\left(g_{\bar{\beta}} N(\beta) \varphi_{s \beta}\right)\right) \\
& =\sum_{\beta \in \bar{Q}_{1}}(-1)^{|\beta|} \operatorname{Tr}\left(\left(N(\beta) \varphi_{s \beta}-\varphi_{e \beta} M(\beta)\right) \circ g_{\bar{\beta}}\right)
\end{aligned}
$$

and

$$
\begin{aligned}
d_{N, M}^{0, *}\left(\left(\epsilon_{\beta}\right)_{\beta \in \bar{Q}_{1}}\right)\left(\left(f_{i}\right)_{i \in Q_{0}}\right) & =\sum_{\beta \in \bar{Q}_{1}} \operatorname{Tr}\left(\epsilon_{\bar{\beta}} \circ\left(M(\beta) f_{s \beta}-f_{e \beta} N(\beta)\right)\right) \\
& =\sum_{i \in Q_{0}} \operatorname{Tr}\left(\left(\sum_{\substack{\beta \in \bar{Q}_{1} \\
e \beta=i}} \epsilon_{\beta} M(\bar{\beta})-\sum_{\substack{\beta \in \bar{Q}_{1} \\
s \beta=i}} N(\bar{\beta}) \epsilon_{\beta}\right) \circ \varphi_{i}\right) .
\end{aligned}
$$

Thus, we have functorially $D \operatorname{Ext}_{\Lambda}^{1}(N, M)=\operatorname{Ker}\left(d_{N, M}^{0, *}\right) / \operatorname{Im}\left(d_{N, M}^{1, *}\right)$. The complexes (8.1) and (8.2) 
are isomorphic:

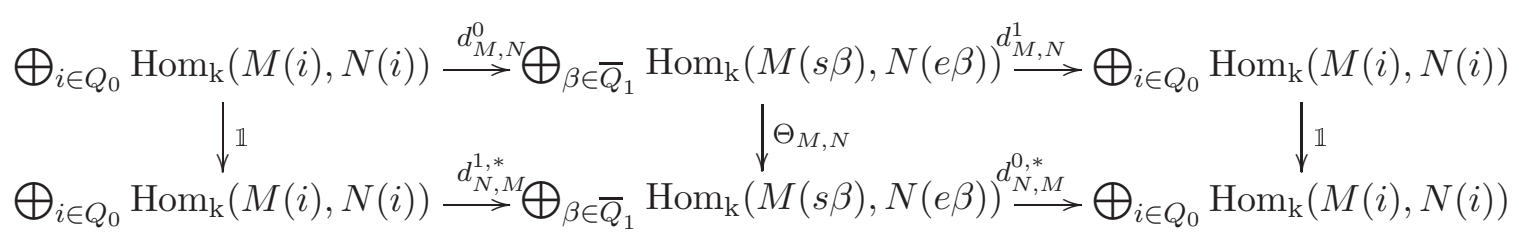

with $\Theta_{M, N}\left(\left(g_{\beta}\right)_{\beta \in \bar{Q}_{1}}\right)=\left((-1)^{|\beta|} g_{\beta}\right)_{\beta \in \bar{Q}_{1}}$. This finishes the proof of Theorem 3 .

\subsection{Remarks}

(1) Assume that $Q$ is connected. Then it is known that in the situation of Lemma 8.1.1 we have an isomorphism of bimodules:

$$
\text { Ker } d^{1} \cong \begin{cases}D \Lambda & \text { if } Q \text { is a Dynkin quiver } \\ 0 & \text { otherwise. }\end{cases}
$$

This follows, for example, from [BBK02, Theorems. 4.8 and 4.9] in the first case and from [BBK02, Proposition 4.2] together with [BK99, Theorem 9.2] in the second case.

Thus, $P^{\bullet}$ is a projective bimodule resolution of $\Lambda$, if $Q$ is not a Dynkin quiver. A similar calculation as in $\S 8.2$ shows that $P^{\bullet}$ is self-dual in the sense of Bocklandt [Boc06, $\S 4.1$ ], thus the bounded derived category $\mathcal{D}^{b}(\Lambda$-mod) is 2-Calabi-Yau [Boc06, Theorem 4.2].

(2) We leave it as an (easy) exercise to derive Crawley-Boevey's formula [Cra00, Lemma 1] and in the non-Dynkin case the equation

$$
\operatorname{dim} \operatorname{Hom}_{\Lambda}(M, N)-\operatorname{dim} \operatorname{Ext}_{\Lambda}^{1}(M, N)+\operatorname{dim} \operatorname{Ext}_{\Lambda}^{2}(M, N)=(\underline{\operatorname{dim}} M, \underline{\operatorname{dim}} N)_{Q},
$$

where $(-,-)_{Q}$ is the symmetric quadratic form associated to $Q$, from the calculations in $\S 8.2$.

\section{ACKNowledGements}

We like to thank Bernhard Keller and William Crawley-Boevey for interesting and helpful discussions. We would like to thank the University of Leeds for one month of hospitality in the summer of 2005 , when most of this work was done.

\section{REFERENCES}

Boc06 R. Bocklandt, Graded Calabi Yau algebras of dimension 3, Preprint (2006), math.RA/0603558.

Bon98 K. Bongartz, Some geometric aspects of representation theory, in Algebras and modules, I, Trondheim, 1996, CMS Conference Proceedings, vol. 23 (American Mathematical Society, Providence, RI, 1998), 1-27.

BBK02 S. Brenner, M. C. R. Butler and A. D. King, Periodic algebras which are almost Koszul, Algebr. Represent. Theory 5 (2002), 331-367.

BK99 M. C. R. Butler and A. D. King, Minimal resolutions of algebras, J. Algebra 212 (1999), 323-362.

CK05 P. Caldero and B. Keller, From triangulated categories to cluster algebras, Preprint (2005), math.RT/0506018, Invent. Math., to appear.

Cra00 W. Crawley-Boevey, On the exceptional fibres of Kleinian singularities, Amer. J. Math. 122 (2000), $1027-1037$.

Dim04 A. Dimca, Sheaves in topology, Universitext (Springer, Berlin, 2004).

GLS05a C. Geiß, B. Leclerc and J. Schröer, Semicanonical bases and preprojective algebras, Ann. Sci. École Norm. Sup. (4) 38 (2005), 193-253. 


\section{SEMicANonicAl BASES AND PREPROJECTIVE ALGEBRAS II}

GLS05b C. Geiß, B. Leclerc and J. Schröer, Auslander algebras and initial seeds for cluster algebras, J. London Math. Soc. (2) 75 (2007), 718-740.

GLS06 C. Geiß, B. Leclerc and J. Schröer, Rigid modules over preprojective algebras, Invent. Math. 165 (2006), 589-632.

Hel60 A. Heller, The loop-space functor in homological algebra, Trans. Amer. Math. Soc. 96 (1960), 382-394.

Lus91 G. Lusztig, Quivers, perverse sheaves, and quantized enveloping algebras, J. Amer. Math. Soc. 4 (1991), 365-421.

Lus00 G. Lusztig, Semicanonical bases arising from enveloping algebras, Adv. Math. 151 (2000), 129-139.

Mac74 R. D. MacPherson, Chern classes for singular algebraic varieties, Ann. of Math. (2) 100 (1974), 423-432.

Rin98 C. M. Ringel, The preprojective algebra of a quiver, in Algebras and modules II, Geiranger, 1966, CMS Conference Proceedings, vol. 24 (American Mathematical Society, Providence, RI, 1998) 467-480,

Ser58 J. P. Serre, Espaces fibrés algébriques, Séminaire C. Chevalley, tome 3, exp. no. 1 (Secrétariat mathématique, Paris, 1958).

Christof Geiss christof@math.unam.mx

Instituto de Matemáticas, Universidad Nacional Autónoma de México, Ciudad Universitaria,

04510 México D.F., México

Bernard Leclerc leclerc@math.unicaen.fr

Laboratoire LMNO, Université de Caen, F-14032 Caen cedex, France

Jan Schröer schroer@math.uni-bonn.de

Mathematisches Institut, Universität Bonn, Beringstrasse 1, 53115 Bonn, Germany 